\title{
Puzzling fluorescent emission from Orion
}

\author{
S. Czesla and J. H. M. M. Schmitt
}

\author{
Hamburger Sternwarte, Universität Hamburg, Gojenbergsweg 112, 21029 Hamburg, Germany \\ e-mail: stefan.czesla@hs.uni-hamburg.de
}

Received 1 April 2010 / Accepted 24 June 2010

\begin{abstract}
Fluorescent X-ray emission offers a rare possibility for studying cool material surrounding active, young stars in the X-ray regime. In this work, we develop a new method to search for fluorescent emission and analyze its temporal behavior, which we apply to a sample of 106 young, active stars in Orion. Our analysis yields a sample of $23 \mathrm{X}$-ray sources with fluorescent emission, including 6 objects already reported on in an earlier study. The fluorescent sources show a wide variety of temporal behavior. While the fluorescent emission is associated with soft X-ray flares in some cases, it sometimes appears as a (quasi) persistent feature, or is seen during truly quiescent periods. We conclude that fluorescent X-ray emission can be observed in a much higher fraction of young, active stars than previously believed. Whether photoionization alone is the excitation mechanism of fluorescent X-ray emission or if electronic collisional excitation also contributes remains debatable. The temporal variability is often hard to reconcile with the photoionization model, which remains plausible if we allow for suitable geometries. Photoionization is preferred to electronic, collisional excitation mainly because the energetics of the latter challenge our current physical understanding.
\end{abstract}

Key words. stars: activity - stars: flare - X-rays: stars

\section{Introduction}

Fluorescent emission pushes the horizon of X-ray astronomy beyond the observation of high-energy material, carrying the imprint of cold matter into the X-ray regime. The origin of fluorescent photons is cool, neutral matter pervaded by ionizing photon or particle radiation coming from a nearby high-energy source. Following an inner shell ionization in the cold matter, excited ions potentially release their energy in the form of fluorescent photons, which convey information on the existence, structure, and composition of their creation site. Geometries appropriate for producing a detectable amount of fluorescent emission are common in a variety of celestial X-ray sources, and fluorescent $\mathrm{X}$-ray emission has been reported in many targets including active galactic nuclei and young stellar objects.

The interaction of an ion with high-energy radiation can end up removing one of the ion's inner shell electrons. In the context of fluorescent emission, two conceivable mechanisms of K-shell electron removal are at issue: photoionization by soft X-rays and collisional excitation by electrons with kinetic energy in excess of the K-shell ionization edge. Independent of the interacting particle, an excited ion returns to a lower energy state through rearrangement of its electron configuration, as a consequence of which the excess energy of the excited state is released by one of two competing processes. Either it is deposited in an "Auger electron", which is expelled from the ion, or it is released in the form of a fluorescent photon. The probability of photon emission rises strongly with increasing atomic number (Bambynek et al. 1972) and is termed the "fluorescence yield". Consulting typical solar system or cosmic abundance patterns (e.g., Anders \& Grevesse 1989) shows that iron becomes the most promising candidate when searching for fluorescent emission. The K-shell ionization energy of neutral iron is $7.112 \mathrm{keV}$, and the energy of its $\mathrm{K} \alpha$ line lies in the $\mathrm{X}$-ray regime at about $6.4 \mathrm{keV}$; both numbers change slowly with increasing ionization (e.g., Kaastra $\&$ Mewe 1993). Spin-orbit interaction and the effects of photon scattering create a characteristic line profile (e.g., Sunyaev \& Churazov 1996), which can, however, hardly be resolved with today's instrumentation in astronomy.

Astronomical detections of the fluorescent $\mathrm{K} \alpha$ line of neutral or weakly ionized iron (in the following "Fe $\mathrm{K}_{\alpha} \mathrm{I}$ line") were first obtained from solar observations (e.g., Neupert et al. 1967; Doschek et al. 1971), in particular, during solar flares. The question of how much photoionization and collisional excitation by electrons contribute to the observed solar fluorescent emission was addressed by several authors. Parmar et al. (1984) analyzed a sample of solar flares and conclude that "most, if not all", of the fluorescent X-rays are due to photoionization and that their results agree well with associated simulations provided by Bai (1979). Even though Zarro et al. (1992) and Emslie et al. (1986) do find evidence for fluorescent photons from electron collisional excitation during the impulsive phase of individual flares, they also agree that the bulk of the fluorescent emission must be attributed to photoionization. The argument for photoionization as the dominant process is based on the strength or equivalent width of the fluorescent line, as well as on its temporal relation to the observed hard and soft X-ray flux.

About thirty years after the reports of fluorescent emission in solar X-ray spectra, Tanaka et al. (1995) published their discovery of the $\mathrm{Fe} \mathrm{K}_{\alpha}$ I line in the spectrum of the active galactic nucleus (AGN) MGC-6-30-15. In excess of an imprint of cold material, this line also carries information on the relativistic space-time structure at its origin, providing deep insight into the inner parts of an AGN. Ballantyne \& Fabian (2003) argue that in AGN sources photoionization is more important than electron or proton collisional excitation, too. The discovery of Tanaka et al. 
intensified the search for the $\mathrm{Fe}_{\alpha} \mathrm{I}$ line in X-ray sources, in the wake of which fluorescent emission was also discovered in several stellar sources other than the Sun.

The stellar sources with reported fluorescent emission include YLWA 16 (Imanishi et al. 2001) with $\mathrm{Fe} \mathrm{K}_{\alpha}$ I line emission detected during a flare, HR 9024, for which Testa et al. (2008) present evidence of the $\mathrm{Fe}_{\alpha}$ I line in a Chandra High Energy Transmission Grating spectrum, and Elias 29, which shows a fluorescent line of variable strength (Giardino et al. 2007). With the exception of HR 9024, the above stars are young stellar objects (YSOs). While photoionization remains the most common explanation for the origin of the fluorescent photons, Giardino et al. (2007) argue that the characteristics of the fluorescent emission observed from Elias 29 are hard to reconcile with the predictions of the photoionization scenario, so they suggest a nonthermal electron population invisible in soft X-rays as a possible explanation for their findings.

As YSOs are known to possess large amounts of cold circumstellar material in the form of disks or envelopes, it seems reasonable to search for the $\mathrm{Fe}_{\alpha} \mathrm{I}$ line in star-forming regions. The Orion Nebula is a center of recent and ongoing star formation, harboring a wealth of YSOs in various evolutionary states. Orion was also the target of a long Chandra observation known as the "Chandra Orion Ultradeep Project" (COUP) (e.g., Getman et al. 2005). Tsujimoto et al. (2005) carried out a search for the $\mathrm{Fe} \mathrm{K}_{\alpha} \mathrm{I}$ line in the COUP data and presented a sample of seven COUP sources with a fluorescent $\mathrm{Fe}_{\alpha} \mathrm{I}$ line detection. One of their sources was further analyzed by Czesla \& Schmitt (2007), who reported the temporal concentration of the observed $\mathrm{Fe} \mathrm{K}_{\alpha} \mathrm{I}$ line flux in the rise phase of one of two flares.

In this study we analyze the X-ray data of 106 COUP sources, including the sample presented by Tsujimoto et al. (2005). We systematically search for fluorescent $\mathrm{Fe} \mathrm{K}_{\alpha} \mathrm{I}$ line emission and present an analysis emphasizing its temporal evolution. Therefore, we first introduce a method for obtaining the light curve of the $\mathrm{Fe}_{\alpha}$ I line feature and discuss its significance as obtained from spectral fitting. We then proceed with an analysis of the $\mathrm{Fe} \mathrm{K}_{\alpha}$ I line behavior in the Tsujimoto et al. (2005) sources and some additional sources with fluorescent emission. Finally, we discuss the impact of our findings on the commonly accepted photoionization model of fluorescent emission and its alternatives.

\section{Detection and analysis of transient spectral features}

Longer integration times lead to the accumulation of signal, allowing for better constrained results unless transient features are under consideration, which are flattened out during longer integrations. Inspired by the problem of analyzing the behavior of the fluorescent Fe $\mathrm{K}_{\alpha}$ I line (Czesla \& Schmitt 2007), we present a systematic method to detect transient spectral features and analyze their temporal variability, often with a higher resolution than allowed for by pure spectral analysis. In the following we apply the relations between several narrow-band light curves to derive the "light curve" of a particular spectral feature.

\subsection{The "line light curve (LLC)" method}

Assume we are given a spectrum with an overall shape described well by some model, $M$, and further, that the overall spectral shape (but not necessarily its normalization) only varies slowly with respect to the temporal resolution of the feature under scrutiny. Our model is supposed to describe the observed spectrum; i.e., it already accounts for our instrument. Below, we assume a thermal model, $M_{\text {th }}$, with an additional emission line, which is, however, not a necessary precondition.

We are interested in the temporal behavior of the emission line, which is supposed to have a central energy of $\epsilon_{\mathrm{e}}$, and is completely covered by the "emission band" $I_{\mathrm{e}}=\left[\epsilon_{\mathrm{e}}-\delta \epsilon_{1}, \epsilon_{\mathrm{e}}+\right.$ $\left.\delta \epsilon_{2}\right]$. The number, $N_{\mathrm{e}}$, of photons expected within this band is given by the sum of the thermal contribution, $N_{\text {th, }}$, given by $M_{\text {th }}$ and the line contribution, $N_{\text {line }}$, such that

$N_{\mathrm{e}}=N_{\text {line }}+N_{\text {th,e }}$.

Now we choose a "comparison band", $I_{\mathrm{c}}=\left[\epsilon_{\mathrm{c} 1}, \epsilon_{\mathrm{c} 2}\right]$, which comprises only continuum emission. In $I_{\mathrm{c}}$ the thermal component provides $N_{\text {th,c }}$ photons, again determined by $M_{\text {th }}$. Defining the "continuum ratio", $r_{\mathrm{c}}$, such that

$N_{\text {th,c }}=r_{\mathrm{c}} \cdot N_{\text {th,e }}$

we obtain the number of expected line photons by

$N_{\text {line }}=N_{\mathrm{e}}-\frac{N_{\text {th,c }}}{r_{\mathrm{c}}}$

For a given energy interval and model, the continuum ratio, $r_{\mathrm{c}}$, is uniquely defined. Narrow-band light curves corresponding to the emission and comparison bands provide both photon numbers on the righthand side of Eq. (3) $\left(N_{\mathrm{e}}\right.$ and $\left.N_{\text {th,c }}\right)$, and, consequently, the lefthand side of Eq. (3) is equal to the line strength as a function of time.

Accordingly, we define the "line light curve (LLC)" as a sequence of pairs, $\left\{t_{i}, l l c_{i}\right\}$, where $l l c_{i}$ denotes the quantity

$l l c_{i}=N_{\mathrm{e}, i}-\frac{N_{\mathrm{th}, \mathrm{c}, i}}{r_{\mathrm{c}, j}}$,

which is basically the number of excess photons in the $i$ th timebin (cf. Eq. (3)), and $t_{i}$ represents the associated time stamp. An extra index, $j$, attached to $r_{\mathrm{c}}$ accounts for those cases where a single value of the continuum ratio is insufficient.

An integration of the LLC over a time span yields a measure of excess emission observed within this span. Formally, we define the "cumulative LLC" (CLLC) as

cllc $c_{n}=\sum_{j=j_{0}}^{j_{0}+n} l l c_{j}$.

With no excess emission, the expectation value of $c l l c_{n}$ is zero by definition, but what is its distribution? Because the values of the summands are statistically determined, the problem of finding the distribution of $\mathrm{cllc}_{n}$ compares to finding the endpoint distribution of a random walk. By definition the quantity $N_{\mathrm{e}, i}$ (see Eq. (4)) obeys a Poisson distribution with an expected value of $\mu_{i}$. If we assume that the quantity $N_{\mathrm{th}, \mathrm{c}, i} / r_{\mathrm{c}, j}$ can be measured very accurately, so that its distribution is adequately approximated by a $\delta$-peak, $\mathrm{d} P\left(N_{\mathrm{th}, \mathrm{c}, i} / r_{\mathrm{c}}\right)=\delta\left(N_{\mathrm{th}, \mathrm{c}, i} / r_{\mathrm{c}}-\mu_{i}\right) \mathrm{d}\left(N_{\mathrm{th}, \mathrm{c}, i} / r_{\mathrm{c}}\right)$ (a justified simplification when $r_{\mathrm{c}}$ is sufficiently large), the distribution in the individual bins, $l l c_{i}$, of the LLC becomes

$P\left(l l c_{i}\right)=\operatorname{Poisson}\left(\mu=\mu_{i}, l l c_{i}+\mu_{i}\right)$,

which is a shifted Poisson distribution with a variance of $\mu_{i}$, but a mean of zero due to the shift, where $l l c_{i}$ may take values in the interval $\left[-\mu_{i}, \infty\right)$.

Equation (6) indicates that the cumulated LLC, $c l l c_{n}$, is a sum of Poisson-distributed quantities, hence Poisson-distributed 
(but shifted) itself. Consequently, the standard deviation of $c l l c_{n}$ becomes

$\sigma\left(\right.$ cllc $\left._{n}\right)=\sqrt{\sum_{i=1}^{n} \mu_{i}}$

This quantity yields an approximation to the expected width of the endpoint distribution of the random walk defined by the numbers obtained from Eq. (5) increasing the upper summation boundary, $n$, step by step.

Combining the estimate of the line strength with that of the underlying continuum, we can also derive an estimate of the $E W$, which is given by

$E W$ [emission band width] $=\frac{N_{\mathrm{e}}-N_{\mathrm{th}} / r_{\mathrm{c}}}{N_{\mathrm{th}} / r_{\mathrm{c}}}=r_{\mathrm{c}} \frac{N_{\mathrm{e}}}{N_{\mathrm{th}}}-1$.

\subsection{The choice of the energy intervals}

For any application of the above method, appropriate emission and comparison energy bands must be found. In the specific case of the fluorescent Fe $\mathrm{K}_{\alpha}$ I line centered at $6.4 \mathrm{keV}$, we argue in favor of the following intervals.

For the emission interval it is convenient to apply $I_{\mathrm{e}}=$ [6.2 keV, $6.5 \mathrm{keV}]$, which, at the spectral resolution provided by the Chandra ACIS and XMM-Newton EPIC instruments, contains $\approx 80 \%$ of all $\mathrm{Fe} \mathrm{K}_{\alpha} \mathrm{I}$ line photons. Unfortunately, the "iron line complex", a number of lines pertaining to Fe XXV and other high ionization stages of iron, is located close to the high-energy boundary of the emission band at about $6.7 \mathrm{keV}$. To avoid contamination, an asymmetric band with respect to the $\mathrm{Fe}_{\alpha} \mathrm{I}$ line center was chosen.

For the comparison interval, $I_{\mathrm{c}}$, we use an energy band extending from $6.2 \mathrm{keV}$ to lower energy. Compared to an interval beyond $6.5 \mathrm{keV}$, typical stellar X-ray spectra provide more photons at lower energies, the detectors onboard Chandra and XMM-Newton are more sensitive here, and, finally, no prominent lines are located there. In practice, we use $5.2 \mathrm{keV}$ as a lower boundary for the comparison interval.

\subsection{The dependences of the continuum ratio on spectral parameters}

At the heart of the LLC approach is the subtraction of the continuum contribution underlying a spectral feature. The continuum has to be extrapolated (or maybe interpolated) from another part of the spectrum, and the "predictor" of its contribution is the continuum ratio, $r_{\mathrm{c}}$. As we are concerned with thermal spectra, a major issue to be addressed is the temperature dependence of $r_{\mathrm{c}}$. Source temperatures do not remain perfectly constant, and we are not able to determine them with arbitrary accuracy. Therefore, it is essential to show that small variations in temperature do not have a serious impact on the continuum ratio.

In Fig. 1 we show the continuum ratio for a wide range of temperatures. The solid and dashed lines indicate the ratio as obtained with and without considering the effect of a varying effective area. In the example we use $6.2-6.5 \mathrm{keV}$ and $5.2-6.2 \mathrm{keV}$ as the boundaries of the emission and comparison bands, and the effective area is modeled with a linear dependence on energy. We use $A_{\text {eff }}\left[\mathrm{cm}^{2}\right]=1000 \mathrm{~cm}^{2}-100 \mathrm{~cm}^{2} / \mathrm{keV} \cdot \epsilon[\mathrm{keV}]$, which in the energy range under consideration is quite representative of numbers obtained for Chandra and XMM-Newton.

Clearly, $r_{\mathrm{c}}$ varies quickly for temperatures below $\approx 2 \mathrm{keV}$ and then approaches an almost constant value. When the effective

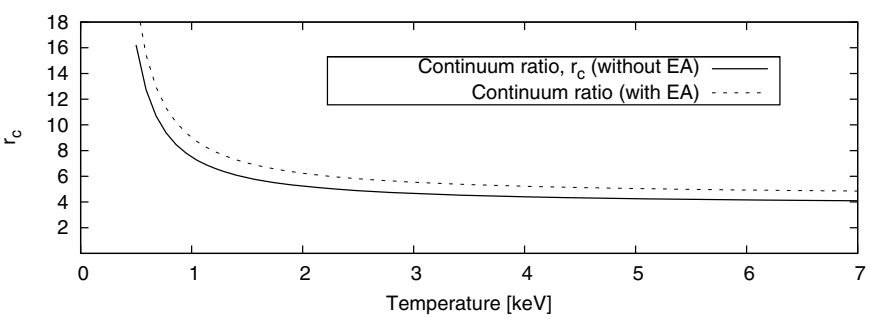

Fig. 1. The continuum ratio, $r_{\mathrm{c}}$, as a function of temperature.

area effect is taken into account, the ratio increases as more photons are detected where the effective area is larger, but the overall behavior remains unaffected. We conclude that $r_{\mathrm{c}}$ is robust against small (and even large) temperature variations for temperatures beyond $2 \mathrm{keV}$.

\section{Spectral binning and the fit statistics}

We are concerned with an individual feature at a well known energy. Therefore, we can adapt our binning approach to meet our purpose best. In particular, we are interested in the narrow $\mathrm{Fe} \mathrm{K}_{\alpha}$ I emission line at $6.4 \mathrm{keV}$. In view of that, we use a fixed bin-size of $\approx 100 \mathrm{eV}$ (actually 7 Chandra channels, which is a little more than $100 \mathrm{eV}$ ) chosen so that one bin is centered on the $\mathrm{Fe} \mathrm{K}_{\alpha} \mathrm{I}$ line energy. For spectral fitting we use the C-statistics (Cash 1979), which can handle low photon numbers per bin, but provides no goodness-of-fit indicator such as $\chi^{2}$ does.

\subsection{The significance of a spectral emission line}

The test for the existence of a spectral line belongs to a category of statistical problems frequently encountered in X-ray astronomy: hypothesis testing in the case of nested models. For a given data set, $D$, we define two alternative models, $m_{1}\left(\boldsymbol{\theta}_{1}\right)$ and $m_{2}\left(\boldsymbol{\theta}_{2}\right)$, specified by their parameter vectors $\boldsymbol{\theta}_{1}$ and $\boldsymbol{\theta}_{2}$ with $n_{1}$ and $n_{2}$ free parameters. Let $n_{2}>n_{1}$ and $m_{1}$ be a subset of $m_{2}$, so that $m_{1}$ is nested within $m_{2}$. The problem consists in deciding which of the models is to be preferred.

In our particular case, $m_{1}$ is a thermal model and $m_{2}$ is the same thermal model extended by a narrow Gaussian line at a fixed energy of $6.4 \mathrm{keV}$. As a measure of the "quality" with which a given parameter vector, $\boldsymbol{\theta}_{1,2}$, reproduces the data, we use the likelihood statistics.

The maximum likelihood achieved with $m_{1}$ is $\mathscr{L}_{1}$, and the fit with the additional narrow spectral line at $6.4 \mathrm{keV}$ provides another likelihood $\mathscr{L}_{2}$. As $m_{1}$ is nested within $m_{2}$, the introduction of the additional component cannot worsen the fit result. A popular tool for finding the preferred model is the likelihoodratio-test (LRT). Protassov et al. (2002), however, pointed out that this test, while being applicable in a variety of other cases, fails in this particular situation, basically because the topology of the underlying parameter space does not fulfill the requirements demanded by the test.

In the case of a narrow emission line, the difference in the dimensions of the parameter spaces $\left(n_{2}-n_{1}\right)$ before and after the introduction of the line is one, and with an appropriate (valid) parameter space, the LRT test statistics, $-2 \log \left(\mathscr{L}_{1} / \mathscr{L}_{2}\right)$, follows a $\chi^{2}$ distribution with a single degree of freedom. This is not the case here, a deficit that cannot be cured by more signal or better instruments, but is inherent in the mathematical structure of the test. Protassov et al. (2002) carried out Monte-Carlo calculations (cf. their Sect. 3.2) and found that in the case of a 


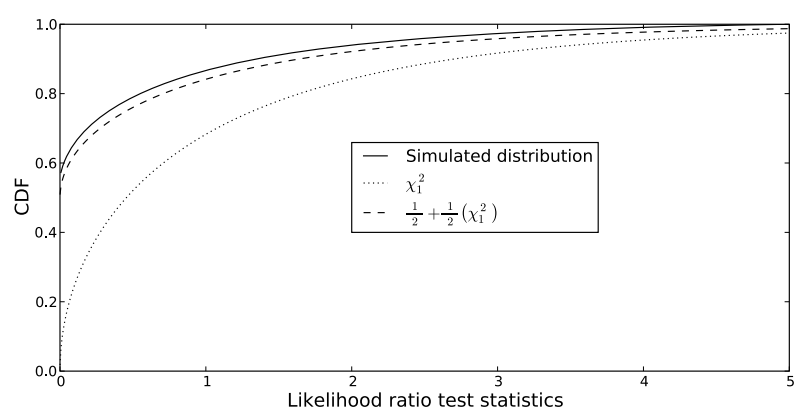

Fig. 2. Distribution of the LRT statistics obtained from Monte Carlo simulations (solid line), the nominal $\chi_{1}^{2}$ distribution (dotted), and the distribution given in Eq. (9) (dashed).

narrow emission line the false-positive rate is considerably overestimated by the $\chi^{2}$ distribution. At the nominal $5 \%$ cut-off of the $\chi^{2}$ distribution the "true" (simulated) value is only $2.6 \%$.

To "calibrate" (cf., Protassov et al. 2002) the LRT statistics for this particular case, we consider the following argument. Assume we are given a continuum model and a narrow line at fixed energy, which may appear as either an emission or an absorption line. In this case, the quantity $-2 \log \left(\mathscr{L}_{1} / \mathscr{L}_{2}\right)$ should, indeed, follow the $\chi_{1}^{2}$ distribution. If we now only allow for one of two possibilities, either emission or absorption, the introduction of the line will not result in any enhancement in the fit statistics where the other possibility is needed. In those cases we obtain $-2 \log \left(\mathscr{L}_{1} / \mathscr{L}_{2}\right)=0$. How often this occurs depends on the form of the distribution underlying the data, which, in our case, is the Poisson distribution. As this distribution is approximately symmetrical around its expectation value, $\mu$, at least for $\mu>1$, about half of the line fits should result in $-2 \log \left(\mathscr{L}_{1} / \mathscr{L}_{2}\right)=0$. In spite of this, the distribution of the likelihood ratio for the emission components remains unaffected if it is present.

Following this argument, the probability of obtaining a value of the LRT statistics, lrt $=-2 \log \left(\mathscr{L}_{1} / \mathscr{L}_{2}\right)$, in the interval $[l r t, l r t+\mathrm{d}(l r t)]$ is given by

$\mathrm{d} P(\operatorname{lr} t)=\frac{1}{2}\left(\delta(l r t)+\chi_{1}^{2}(l r t)\right) \mathrm{d}(l r t)$,

where $\delta(l r t)$ denotes the $\delta$-distribution. As described above half of the distribution "is glued to 0" and the other half follows a $\chi_{1}^{2}$ distribution.

To corroborate the validity of our reasoning, we carried out Monte Carlo simulations similar to those of Protassov et al. (2002). We simulate a number of observations using a constant continuum model and Poisson statistics. First, we fit the normalization yielding a likelihood of $\mathscr{L}_{1}$, and, second, we fit both the continuum normalization and the line normalization simultaneously to the same observation. For every simulated observation, we record the value of the LRT statistics, $-2 \log \left(\mathscr{L}_{1} / \mathscr{L}_{2}\right)$.

The results of the Monte Carlo simulations with a continuum expectation value of 2 photons per bin are shown in Fig. 2. Clearly, the nominal $\chi^{2}$ distribution with a single degree of freedom fails to reproduce the simulated findings, whereas the distribution given in Eq. (9) provides a considerably better approximation.

Therefore, when testing for the presence of a narrow emission line, we can interpret the numbers obtained for the LRT statistics using Eq. (9) as a reference distribution. In practice, this means that our sensitivity is approximately twice as high as a blind application of the LRT implies.
Table 1. The COUP observations.

\begin{tabular}{llll}
\hline \hline Obs. ID & Start $[\mathrm{ks}]^{1}$ & Length $[\mathrm{ks}]$ & Identifier \\
\hline 4395 & 0 & 100 & O1 \\
3744 & 156 & 164 & O2 \\
4373 & 384 & 172 & O3 \\
4374 & 616 & 169 & O4 \\
4396 & 841 & 165 & O5 \\
3498 & 1070 & 69 & O6 \\
\hline
\end{tabular}

Notes. ${ }^{(1)}$ Counted from MJD 52647.9.

\subsection{Significance estimates in time-resolved spectroscopy}

Let us assume an observation of length $T$ to be at our disposal, and in the data we detect a source, which is only slightly variable on the time scale $T$. Likewise, we choose an arbitrary time interval of length $t$ so that $t \ll T$ and carry out a spectral analysis, using only the data pertaining to that sub-interval. Our analysis leaves us with an emission line to which we can assign a high probability, $p_{\mathrm{e}}$, for being set apart from the noise along the lines of the LRT presented in the previous section. Now we ask for the probability of finding an emission line with the same significance in any of the time intervals of length $t-$ let there be an integer number $N \approx T / t$ - contained in our observation under the assumption that no emission line is present.

This set-up can be interpreted as a "Bernoulli experiment" with its probability of success given by the probability for a spurious detection, $1-p_{\mathrm{e}}$. Therefore, the chance, $p_{\mathrm{s}}$, to obtain one (or more) such emission line(s) from the analysis of any of the $N$ subintervals by pure chance amounts to

$p_{\mathrm{s}}=1-p_{\mathrm{e}}^{N}$,

which must be interpreted as the "true" significance of our detection.

The main restriction above is that the source may only be slightly variable. While this assumption is essential for interpretation as a Bernoulli experiment, it is known to be invalid for many X-ray sources. For example a strong flare may provide the majority of the source photons and also $\mathrm{Fe} \mathrm{K}_{\alpha}$ I line photons, while still being short. In such a case, Eq. (10) no longer provides a meaningful estimate of the significance. To obtain a meaningful number in these cases, we propose to use $N=N_{\text {tot }} / N_{\text {int }}$ in Eq. (10), where $N_{\text {tot }}$ is the total number of observed source photons in an appropriate band and $N_{\text {int }}$ is the number of photons in the interval under consideration.

If the source is constant both approaches are equivalent and mathematically correct. In the case of a variable source, none of the above approaches is strictly correct. Nonetheless, we use Eq. (10) to obtain significance estimators, accounting for the choice of a particular fraction of the data.

\section{Data analysis}

The Orion Nebular Cloud is among the best-studied star-forming regions. In particular, it was the target of a large Chandra program aimed at uncovering the X-ray properties of young stars the Chandra Orion Ultra Deep Project (COUP). The COUP campaign consists of six consecutive observations of Orion providing a total observation time of $\approx 840 \mathrm{ks}$ distributed over nearly 13 days, which corresponds to a time coverage fraction of $\approx 75 \%$.

In Table 1 we summarize the basic information on the COUP observations and refer the reader to Getman et al. (2005) for a more detailed discussion. The last column in Table 1 gives the identifier we use throughout this work to refer to the individual observations. 


\subsection{Defining the search sample}

The entire COUP source sample comprises 1616 X-ray sources (Getman et al. 2005). From these sources, we extracted the subsample used in our search for the fluorescent $\mathrm{Fe} \mathrm{K}_{\alpha} \mathrm{I}$ line. Since the line has a fixed energy of $6.4 \mathrm{keV}$, it is advisable to only include those sources that show a sufficient number of counts in an appropriate energy interval around $6.4 \mathrm{keV}$. In our analysis we used only sources with more than 30 counts in the "emission-band" (6.2-6.5 keV). In particular, we iterated through the sources presented by Getman et al. (2005), extracted the source counts in the emission band, corrected them for the PSF coverage of our extraction region, and included the source when our search criterion was matched. For the weakest source included in the sample of Tsujimoto et al. (2005) (COUP 649), we find 31 emission band photons, making our sample include the Tsujimoto et al. sources as a subsample.

Our final sample comprises a total of 108 COUP sources, which provide the the most promising candidates for our search for fluorescent emission. For our purpose, COUP 123 and COUP 124 can be treated as a single source, because they are too close to be resolved ( $1.4 \mathrm{arcsec}, \approx 5 \operatorname{arcmin}$ off-axis). Their spectrum is appropriately described by a single temperature thermal model, which indicates that both may, indeed, refer to the same X-ray source. Additionally, we had to exclude COUP 90 and COUP 828 from our sample, because they are located on chip edges, which prevented us from obtaining appropriate spectra (CIAO 4.1), so that the sample size reduces to 106 COUP sources.

\subsection{Extraction regions and pile-up}

For most of our sources we used circular extraction regions covering the entire source. Our search sample necessarily contained the most X-ray luminous sources in the COUP field, which may suffer from severe pile-up. To avoid a strong pile-up effect, we used annulus shaped extraction regions in 29 cases. Whether pile-up becomes severe depends not only on the source count rate, but also on its temporal behavior and positioning on the detector. We chose the annulus parameters in accordance with these considerations and note whenever an annulus shaped region was used for extraction.

\subsection{A standard analysis}

Our search sample consists of 106 sources. To achieve a homogeneous scan for the fluorescent $\mathrm{Fe} \mathrm{K}_{\alpha}$ I line, they should be treated with standard criteria, eliminating biases arising from individual, subjective choices during the analysis.

\subsubsection{Bayesian block analysis of the broad-band light curves}

A key ingredient in the LLC method is determining of the correct continuum ratio. The observed count rate for individual X-ray sources can vary by orders of magnitude during the COUP campaign. Although the continuum ratio is relatively robust against temperature variations (see Sect. 2.3), it is advisable to distinguish between periods of different count rate regimes, possibly indicating different source spectra and continuum ratios. Of course a transition between different spectra may not always be accompanied by a count rate change, but we neglect this possibility here.

Our count rate analysis is based on the broad-band (2-9 keV) light curve. To separate the entire campaign data of individual sources into intervals with comparable count rate, we applied the concept of "Bayesian blocks" elaborated by Scargle (1998). The method of Bayesian blocks "converts raw photon counts into the most probable piecewise constant representation of brightness as a function of time" (Scargle 1998).

We applied an iterative algorithm to find individual change points in the light curves. According to Scargle (1998), this does not represent the exact mathematical treatment of the problem, but, nonetheless, yields comparable results. As pointed out by Scargle, an appropriate condition must be found to stop the iteration before segmenting the data set excessively. Instead of applying the Bayes factor (Eq. (48) in Scargle 1998) and a prior, we used the likelihood ratio, which can be easily calculated, to decide whether to introduce a change point or not.

As the continuum ratio only weakly depends on the spectral temperature, we aimed at a coarse segmentation of the light curves in order to separate periods between which the spectrum of the associated source may significantly differ and with it the continuum ratio. To introduce a change point, we demanded no less than 100 counts per block, a minimum block length of $20 \mathrm{ks}$, and a value of the LRT statistics over 100.

With this method we separated the 106 source light curves into 419 intervals with comparable count rates, and proceeded by generating spectra for every individual source and block, which can be used to determine the corresponding continuum ratio.

\subsubsection{Automated model fits}

For our analysis we used both thermal and power-law models, for which we set up automated fit procedures using XSPEC. The fits of the thermal model are based on the $0.3-9 \mathrm{keV}$ energy band. As a basis for our fits, we used an absorbed twotemperature APEC (e.g. Smith et al. 2001) model extended by an emission line representing the fluorescent $\mathrm{Fe}_{\alpha} \mathrm{I}$ line at $6.4 \mathrm{keV}$. Additionally, we allowed for emission lines at various other energies, which are later used to substantiate our results. The nominal intrinsic width of all lines is $0 \mathrm{eV}$. All element abundances but that of iron were treated as a single fit-parameter, which was also shared among the two thermal components. In contrast, the iron abundance represents an independent fit-parameter in both thermal components.

Starting a fit process with unfavorable initial conditions can easily lead to unphysical results, even if the fit converges appropriately. Therefore, the process must be set up carefully to avoid converging to unrealistic solutions. Our approach is the following. We start using only one of the two available thermal components and then add in the second. If the enhancement in C-statistics exceeds 6.4 by introducing the later component, this solution is kept, otherwise the one temperature solution is used. The value of 6.4 corresponds to the $90 \%$ cut-off in a likelihood ratio test comparing models that differ by 3 degrees of freedom, which are temperature, iron abundance, and normalization here. The numerical equality with the fluorescent line energy is purely accidental.

Furthermore, we apply a number of restrictions to the temperatures and abundances to avoid unphysical solutions. If the temperature of any thermal component exceeds $8 \mathrm{keV}$, we fix it at $8 \mathrm{keV}$ and reiterate the fit, because temperatures beyond this limit can hardly be resolved in Chandra ACIS I spectra. If one of the iron abundances exceeds 10 times the solar value, it is fixed to the value of the global abundance pattern, because it is usually not constrained well in this case. Similarly, the global abundances are fixed at solar values, if the fit result exceeds 10 times the solar value. 
After an acceptable solution was found, we obtained fluxes and equivalent widths for the fluorescent line and the comparison line on the basis of these fits. Finally, the fits were used to derive the continuum ratio for the Bayesian block under consideration. In the same vein, effective area and absorption were automatically accounted for in its derivation.

To complement the results of the thermal model, we also adapted an absorbed power-law model using only the high energy tail (3-9 keV band) of the spectra. This model was extended by narrow emission lines accounting for the $\mathrm{Fe} \mathrm{K}_{\alpha} \mathrm{I}$ line at $6.4 \mathrm{keV}$ and the hot iron line complex at $6.7 \mathrm{keV}$. The results of these fits are particularly interesting as they yield the $E W$ of the hot iron line complex, and they provide means to check whether the adaption of the thermal model failed.

\subsubsection{Line light curve analysis}

As soon as the continuum ratios are determined, the LLC and the CLLC can be calculated. According to our reasoning, periods of bona fide fluorescent emission are characterized by an increase in the CLLC. Taking an impartial position, the morphology of the increase is unknown. We can neither say on what time scales the CLLC typically evolves, nor say whether the rise is continuous or not, nor even whether it is correlated with the soft X-ray continuum.

In our search for candidate intervals, we first exclude those sources that do not show a larger CLLC excess than the expected one (cf. Eq. (7)). For each remaining source, we search all conceivable connected intervals longer than $20 \mathrm{ks}$ for the largest relative excess in fluorescent photons. About 4000 of such intervals can be constructed considering that we have 89 bins of $\approx 10 \mathrm{ks}$ length each. For each of these conceivable intervals, we calculate the quantity $N_{\sigma}$ defined by

$N_{\sigma}=\frac{\sum_{i} L L C_{i}}{\sqrt{\sum_{i} \frac{N_{\mathrm{c}, i}(\Delta t)_{i}}{r_{\mathrm{c}, i}}}}$,

where $i$ is an index running over all light curve bins in the interval, $N_{\mathrm{c}}$ is the observed rate of comparison band photons, $r_{\mathrm{c}}$ the continuum ratio, and $\Delta t$ the duration of the individual bins. As an additional constraint, we demanded that each interval to be considered must at least comprise 5 CLLC excess photons and $\geq 50$ comparison band photons to be relevant.

The most promising candidate interval is the one with the highest $N_{\sigma}$ value. Nevertheless, as every source will contain one such interval, we have to choose a reasonable limit for $N_{\sigma}$; only beyond this limit intervals shall valid candidate intervals be considered. Replacing the Poisson distributions by normal distributions, it may be shown that $N_{\sigma}$ approximately follows a standard normal distribution. Therefore, the probability that $\max \left(N_{\sigma}\right)$ exceeds a limit, $L$, becomes

$p\left(\max \left(N_{\sigma}\right)>L\right)=1-\operatorname{Normal}_{0,1}\left(N_{\sigma}<L\right)^{n}$,

where $n$ is the number of intervals for which $N_{\sigma}$ is determined and Normal $_{0,1}$ denotes the standard normal distribution. If, as in our case, $n$ is $\approx 4000$ and we demand $p\left(\max \left(N_{\sigma}\right)>L\right)=0.2$, we obtain $L \approx 3.9$, which is considered a reasonable limit for $N_{\sigma}$. The preceding approximation does not take into account that most intervals overlap and are, therefore, not independent, and it also neglects, that some may not contain enough photons to be relevant. To overcome these shortcomings, we obtained a more appropriate limit by a Monte Carlo simulation. For each source, we simulated a number of light curves based on Poisson statistics with the observation as our expectation value. From these artificial light curves, a distribution of $\max \left(N_{\sigma}\right)$ can be derived, which takes all constraints into account; however, these results are in good agreement with the approximation.

Following this approach, we found candidate intervals in 25 of our sample sources, and we proceeded by extracting the associated spectra. We then applied the same automated thermal fits as above to find a spectral model. On the basis of this model, we calculated the significance of the fluorescent $\mathrm{Fe} \mathrm{K}_{\alpha} \mathrm{I}$ line in the interval using Eq. (9) and applied Eq. (10) to account for our interval choice. We evaluated Eq. (10) using both definitions of the number of repetitions in the Bernoulli experiment, i.e., interval length and the number of enclosed photons. The maximum of both results was than used as a significance estimator. We prefer the maximum to the more conservative minimum here, because the latter would necessarily underestimate the significance during a flare, which is typically short but intense. We also note that the application of Eq. (10) is no longer fully justified here, because the "experiments" are not fully independent when a distinguished interval is chosen in advance. Nonetheless, the number is used as an estimate.

In Table 2 we list those sources with a line detection at a significance level of $\geq 95 \%$. Our sample comprises 23 sources including 6 sources already reported on by Tsujimoto et al. (2005). We excluded COUP 554 from the list, because a correct analysis of this source was impeded by pile-up. When consulting the $95 \%$ limit, we estimate that $1-2$ detection given in Table 2 must be attributed to noise. Considering the difficulties in defining the true significance of the $\mathrm{Fe}_{\alpha}$ I line in this temporally resolved analysis, we note that also accepting 3-4 spurious detections, does not seriously alter the outcome of the analysis.

\section{Discussion}

This section starts with a presentation of individual sources, proceeds with an analysis of several sample properties, and finally closes with a discussion of the physical scenario of $\mathrm{Fe} \mathrm{K}_{\alpha} \mathrm{I}$ line creation in light of our results.

\subsection{Previously known COUP sources with fluorescent emission}

Tsujimoto et al. (2005) present a sample of 7 COUP sources (COUP sequence numbers 331, 561, 621, 647, 649, 1030, and 1040) with a detection of fluorescent emission. The authors, however, did not carry out time-resolved spectroscopy, but used time-averaged spectra. Below we present the outcomes obtained for the Tsujimoto et al. sources to demonstrate the techniques developed so far. In the following, values given in parentheses refer to the $90 \%$ confidence range for the given parameter.

\subsubsection{COUP $331-\mathrm{V} 1486$ Ori}

This source was already discussed in detail in a previous publication by Czesla \& Schmitt (2007). V 1486 Ori shows two major flare events during the COUP campaign. The first, rather short one, takes place towards the end of O1 ("Flare I" in Czesla \& Schmitt 2007), and no significant fluorescent $\mathrm{Fe} \mathrm{K}_{\alpha} \mathrm{I}$ line emission was detected here. In Fig. 3 (panel A) we show the broadband light curve, the change points returned by the Bayesian analysis, and the resulting CLLC. The first flare is not accompanied by a considerable excess in the CLLC. The spectrum is well-fitted by an absorbed thermal model with a strong (hot) iron line complex at $\approx 6.7 \mathrm{keV}$. 
S. Czesla and J. H. M. M. Schmitt: Puzzling fluorescent emission from Orion
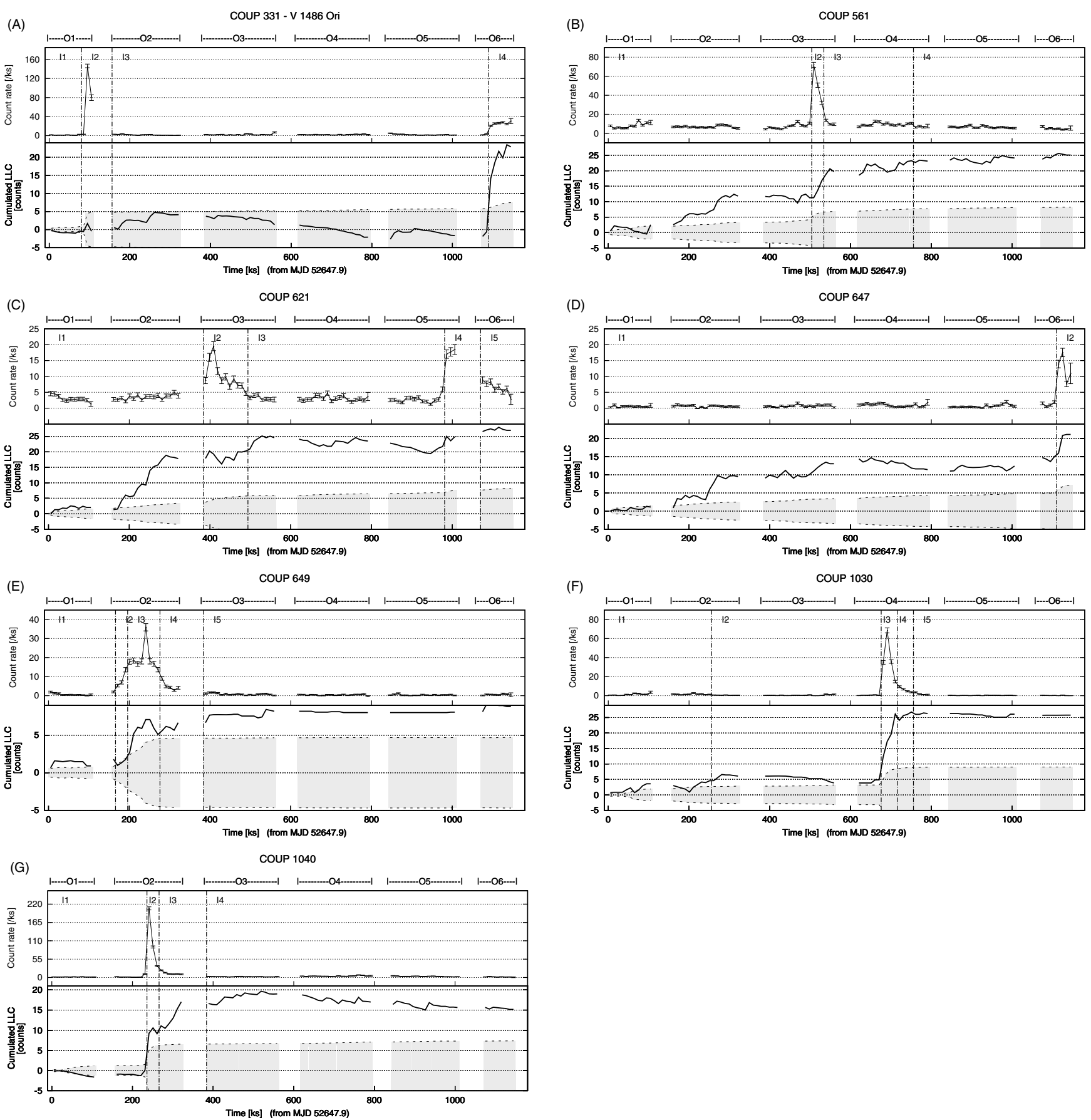

Fig. 3. Panel A) to G): light curves for the objects reported on by Tsujimoto et al. (2005). The time axis refers to the begin of the COUP campaign (MJD 52 647.87). Upper panel: broad-band (2-9 keV) light curve (BBLC). Lower panel: the cumulated LLC (CLLC) and an estimate of its width (gray shaded, see Eq. (7)). Vertical separators mark change points associated with a shift of the activity level (determined by the Bayesian block analysis of the BBLC).

The CLLC clearly indicates that the fluorescent $\mathrm{Fe} \mathrm{K}_{\alpha} \mathrm{I}$ line emission is present during the rise phase of a second, more violent flare ignited during O6, which is in line with previous findings (Czesla \& Schmitt 2007). The automated search for intervals with fluorescent $\mathrm{Fe}_{\alpha}$ I emission returned the 1080-1110 ks period (cf. Table 2) as the most promising candidate interval. In Fig. 4 we present the LLC and the spectrum for the corresponding part of the observation. The LLC clearly shows the concentration of $\mathrm{Fe}_{\alpha} \mathrm{I}$ line emission in the rise phase of the flare, and the spectral analysis yields an $E W$ of 1440(960-1930) eV for the line.
The measured $E W$ of this $\mathrm{Fe} \mathrm{K}_{\alpha} \mathrm{I}$ line exceeds typical model predictions (see Sect. 6) by an order of magnitude, so it is particularly interesting. A more detailed discussion about the origin of this $\mathrm{Fe} \mathrm{K}_{\alpha} \mathrm{I}$ line is given later in Sect. 6.3.

\subsubsection{COUP 561}

Figure 3B shows the results of our light curve analysis for COUP 561. The CLLC shows two phases of increase. During the second COUP observation, as in the longest part of the COUP 
Table 2. Most promising intervals for fluorescent emission and basic fit results.

\begin{tabular}{|c|c|c|c|c|c|c|c|c|}
\hline $\begin{array}{l}\operatorname{COUP}^{a b} \\
\text { seq. no. }\end{array}$ & $N_{\sigma}$ & $\begin{array}{l}\text { Interval } \\
{[\mathrm{ks}]}\end{array}$ & $\begin{array}{l}\text { Sign. } \\
{[\%]}\end{array}$ & $\begin{array}{l}\mathrm{Fe} \mathrm{K} \mathrm{K}_{\alpha} \mathrm{I} E W \\
{[\mathrm{eV}]}\end{array}$ & $\begin{array}{l}N_{6.4 \mathrm{keV}} \\
{\left[10^{-7} \mathrm{ph}\left(\mathrm{cm}^{2} \mathrm{~s}\right)^{-1}\right]}\end{array}$ & $\begin{array}{l}N_{\mathrm{H}} \\
{\left[10^{22} \mathrm{H} \mathrm{cm}^{-2}\right]}\end{array}$ & $\begin{array}{l}T_{1} \\
{[\mathrm{keV}]}\end{array}$ & $\begin{array}{l}T_{2} \\
{[\mathrm{keV}]}\end{array}$ \\
\hline 7 & 3.95 & $60-961$ & 99.99 & $446(216-667)$ & $2.48(1.20-3.71)$ & $0.02(0.01-0.03)$ & $2.08(1.97-2.34)$ & $0.83(0.81-0.85)$ \\
\hline 9 & 3.86 & $514-851$ & 99.35 & $366(123-628)$ & $7.95(2.67-13.63)$ & $0.19(0.15-0.22)$ & $3.72(3.12-5.14)$ & $1.00(0.89-1.07)$ \\
\hline $245^{a}$ & 5.32 & $80-891$ & 99.98 & $482(261-906)$ & $.90(1.03-3.57)$ & $0.16(0.10-0.20)$ & $4.51(3.60-8.23)$ & $0.84(0.77-1.00)$ \\
\hline 267 & 4.81 & 30-196 & 99.88 & $400(176-672)$ & $13.02(5.73-21.88)$ & $2.42(2.15-2.61)$ & 8.00 & - \\
\hline $331^{b}$ & 6.92 & $1080-1110$ & 100 & 1437(959-1927) & $78.9(52.7-105.8)$ & $1.79(1.60-2.00)$ & 8.00 & - \\
\hline 449 & 3.67 & 296-961 & 98.69 & $212(60-391)$ & $1.62(0.46-2.98)$ & $1.53(1.29-1.76)$ & $2.87(2.41-3.47)$ & $0.98(0.76-1.13)$ \\
\hline $561^{b}$ & 4.64 & $100-414$ & 99.98 & $569(279-914)$ & $4.60(2.25-7.38)$ & $0.98(0.90-1.07)$ & $2.40(2.20-2.64)$ & - \\
\hline 599 & 4.65 & $10-1080$ & 99.99 & $448(216-663)$ & $2.07(1.00$ & 7.64(6.6 & $3.67(2.86-4.89)$ & - \\
\hline $621^{b}$ & 6.21 & 10-296 & 99.97 & $602(307-973)$ & $4.84(2.47-7.83)$ & $4.54(4.29-5.24)$ & $3.25(2.64-4.98)$ & $0.08(0.00-0.09)$ \\
\hline $647^{b}$ & 4.22 & $50-626$ & 99.85 & $440(194-767)$ & $3.42(1.51-5.96)$ & $25.23(20.33-31.63)$ & $5.87(3.69-9.32)$ & - \\
\hline 662 & 4.09 & $246-394$ & 98.35 & $206(93-434)$ & $12.61(5.70-26.57)$ & $16.06(14.40-17.88)$ & 8.00 & $0.08(0.00-0.11)$ \\
\hline 669 & 4.39 & $384-776$ & 99.88 & $384(160-616)$ & $2.73(1.14-4.38)$ & $0.33(0.29-0.36)$ & 8.00 & $1.02(0.95-1.06)$ \\
\hline 680 & 4.21 & $80-716$ & 99.94 & $410(190-689)$ & $2.25(1.04-3.77)$ & $11.63(9.68-13.41)$ & 8.00 & $0.08(0.00-0.09)$ \\
\hline 682 & 3.53 & $851-1130$ & 98.62 & $312(108-591)$ & $3.01(1.04-5.71)$ & $1.52(1.22-1.76)$ & $3.05(2.71-3.71)$ & $0.09(0.00-0.11)$ \\
\hline $797^{a}$ & 3.26 & $20-951$ & 96.85 & $82(11-151)$ & $3.28(0.45-6.08)$ & $29.04(27.23-30.92)$ & 8.00 & - \\
\hline 801 & 4.79 & $166-636$ & 99.54 & $456(163-785)$ & $5.91(2.11-10.20)$ & $0.41(0.36-0.48)$ & $3.08(2.40-4.25)$ & $1.04(0.89-1.23)$ \\
\hline 942 & 3.90 & $286-716$ & 99.99 & $308(173-506)$ & $4.68(2.62-7.69)$ & $1.08(0.99-1.15)$ & $3.44(3.16-3.87)$ & - \\
\hline $1030^{a, b}$ & 3.17 & $206-716$ & 99.68 & $143(56-231)$ & $6.35(2.50-10.25)$ & $16.14(13.85-18.68)$ & 8.00 & $0.28(0.22-0.33)$ \\
\hline 1035 & 4.09 & $394-686$ & 98.26 & $410(153-851)$ & $2.78(1.04-5.77)$ & $1.76(1.59-2.02)$ & $2.24(1.92-2.53)$ & - \\
\hline $1040^{b}$ & 3.80 & $266-544$ & 98.62 & $307(110-664)$ & $3.14(1.12-6.78)$ & $1.44(1.29-1.60)$ & $2.95(2.58-3.46)$ & - \\
\hline 1080 & 4.35 & $766-961$ & 98.58 & $332(107-578)$ & $6.99(2.26-12.19)$ & $2.35(1.95-2.86)$ & $3.28(2.68-3.86)$ & $0.63(0.00-0.88)$ \\
\hline 1380 & 3.95 & $554-786$ & 96.37 & 187(49-336) & $5.68(1.49-10.21)$ & $0.51(0.45-0.59)$ & 8.00 & $1.15(0.93-1.47)$ \\
\hline 1499 & 4.50 & $384-524$ & 99.69 & $372(145-631)$ & $7.25(2.83-12.30)$ & $0.43(0.37-0.49)$ & $4.56(3.88-5.40)$ & - \\
\hline
\end{tabular}

Notes. ${ }^{(a)}$ Annulus shaped source region; ${ }^{(b)}$ Sources present in the sample of Tsujimoto et al. (2005).
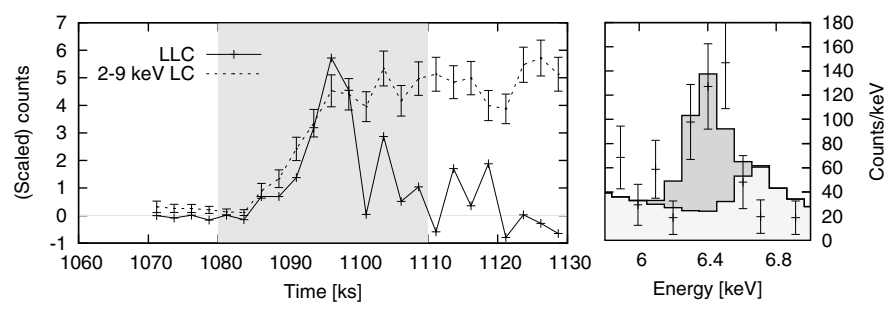

Fig. 4. Left: LLC and (scaled) broad-band light curve (2.5 ks binning) for COUP 331 (O6). Right: excerpt of the spectrum (corresponding to the gray-shaded time span in the left panel). Both a model with and one without extra line emission are indicated.
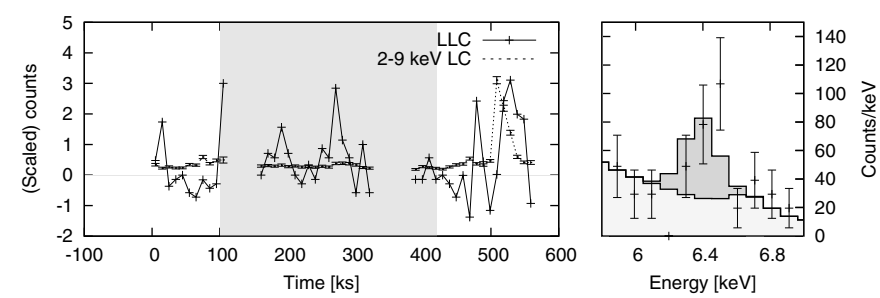

Fig. 5. Left panel: the LLC and the broad-band light curve (both $10 \mathrm{ks}$ binning) of COUP 561. The latter is scaled to show the same maximal count rate as the LLC. The gray-shaded area denotes the time span for which an excerpt of the spectrum is shown in the right panel. There we show the X-ray spectrum around $6.4 \mathrm{keV}$ and indicate both a thermal model with and one without excess emission at the energy of the fluorescent $\mathrm{Fe} \mathrm{K}_{\alpha} \mathrm{I}$ line.

campaign, COUP 561 is encountered in a quiescent state to judge from the BBLC. Nonetheless, the CLLC indicates about 10 excess photons in the emission band.

In Fig. 5 (left panel) we show the LLC pertaining the first half of the COUP campaign and the most promising candidate
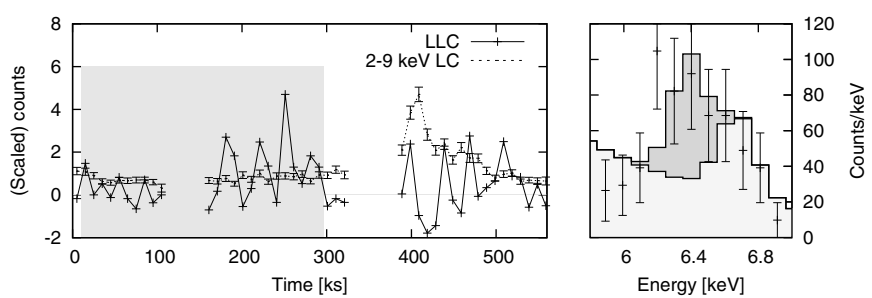

Fig. 6. COUP 621, as in Fig. 5.

interval (100-414 ks) for fluorescent emission. In the right panel we plot the spectrum obtained for this interval, which shows a fluorescent line with an $E W$ of $570(280-910) \mathrm{eV}$. The hot ironline complex is virtually absent.

During the flare in the following observation, the CLLC indicates another increase of about 10 photons. This number is confirmed by a spectral analysis of the associated span (504-544 ks), where we find an $\mathrm{Fe} \mathrm{K}_{\alpha} \mathrm{I}$ line with an $E W$ of 132(12-270) eV. Because of the larger flare continuum, the line is much less prominent here, and with $96 \%$ it is also much less significant.

\subsubsection{COUP 621}

The outcome of the light curve analysis for COUP 621 is shown in Fig. 3C. COUP 621 shows two flares with the first one fully covered by the observation (except for a probably small fraction of the rise-phase) and the second one remaining unobserved for $\approx 60 \mathrm{ks}$ of its central phase. None of these flares is accompanied by a CLLC excess. The CLLC shows an increase that is not associated with one of the flares, but instead emerges during a period of quiescence to judge from the BBLC.

In Fig. 6 we show the LLC corresponding to $\mathrm{O} 2$ and $\mathrm{O} 3$ in the left panel where we also indicate the most promising interval 

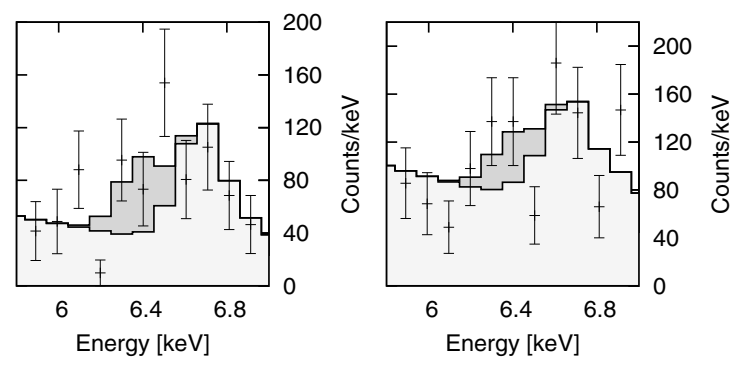

Fig. 7. Left panel: excerpt of the spectrum during the bona fide quiescent phase of COUP 647. Right panel: the same part of the spectrum during the flare.

(cf. Table 2). In the right panel we show the associated spectrum. Excess emission at the location of the fluorescent line is prominent with a line $E W$ of $600(310-970) \mathrm{eV}$, and the hot iron line complex is weak compared to it.

This case is special because two flares detected in soft X-rays are not accompanied by fluorescent $\mathrm{Fe}_{\alpha} \mathrm{K}_{\alpha}$ I line emission, while there is a line detection during a period preceding the first flare. The line emission may be attributed to a hidden flare located at a geometrically quite favorable position. At least it must be attributed to an event that does not show up in soft X-rays.

\subsubsection{COUP 647}

Among the sources analyzed by Tsujimoto et al., COUP 647 is by far the most deeply embedded one with a hydrogen column density of $\approx 31 \times 10^{22} \mathrm{~cm}^{-2}$. The CLLC shows two phases of increase. One associated with a flare towards the end of the COUP campaign and another one earlier in a presumably quiescent phase of the source (see Fig. 3D). Our automated CLLC analysis returns the first phase, in particular the 50-626 ks interval, as the most promising candidate interval, for which we show the spectrum in the left hand panel of Fig. 7. The $E W$ of the line in this phase is 440(194-767) eV. Additionally, we show the spectrum of the flare during which the line shows up with an $E W$ of $150(10-300) \mathrm{eV}$.

\subsubsection{COUP 649}

Among the sources of fluorescent emission reported on by Tsujimoto et al. (2005), COUP 649 is the one with the weakest $\mathrm{Fe} \mathrm{K}_{\alpha} \mathrm{I}$ line. Figure $3 \mathrm{E}$ shows the BBLC and the associated CLLC. The source signal is dominated by a single flare, beyond which the source is virtually invisible. This source was rejected by our automated analysis, because the determined excess is not sufficient to fulfill our criteria. The light curve of COUP 649 shows rapid variability where the source is visible, and we speculate that this impedes an appropriate light curve analysis.

In a spectral analysis of the flare, however, we find a fluorescent emission with an $E W$ of $\approx 260(80-450) \mathrm{eV}$, compatible with the results given by Tsujimoto et al. (2005). In this case, the $E W$ is not affected by time averaging, because only the flare contributes signal.

\subsubsection{COUP 1030}

Figure $3 \mathrm{~F}$ shows the light curves pertaining to COUP 1030. A flare occurs in $\mathrm{O} 4$, and we find that the source is piled-up in this state. In the following analysis we, therefore, revert to an

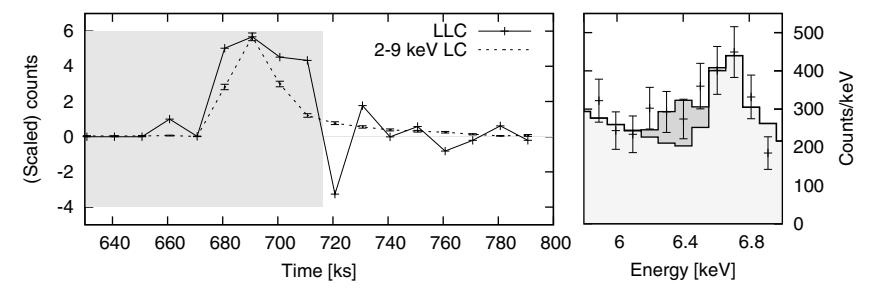

Fig. 8. COUP 1030, as in Fig. 5.
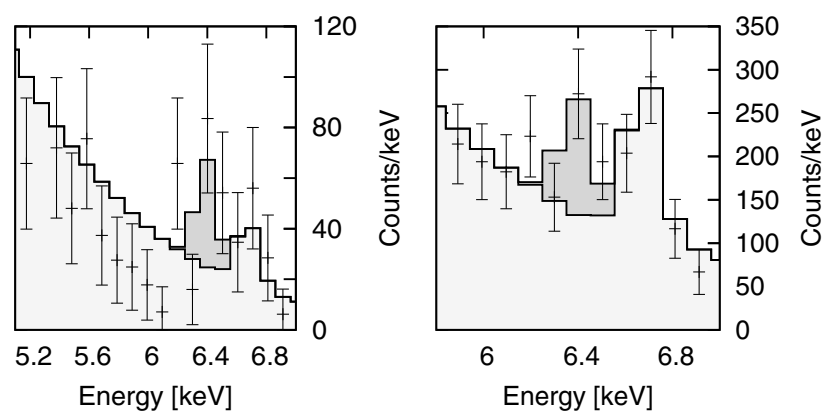

Fig. 9. Left panel: the spectrum during the automatically determined, most promising fluorescent interval of COUP 1040. Right panel: the spectrum during the second COUP observation $(\mathrm{O} 2)$.

annulus-shaped source region neglecting the innermost 0.6 arcsec.

The automated CLLC analysis yields the 206-716 ks interval as the most promising for detecting fluorescent $\mathrm{Fe} \mathrm{K}_{\alpha}$ I emission. This interval covers the flare, which, according to the CLLC, provides the largest contribution of fluorescent photons. In the right hand panel of Fig. 8, we show the spectrum of COUP 1030 during the most promising interval, and our spectral analysis shows a fluorescent line with an $E W$ of 143(56-231) eV. The result is compatible with the values given by Tsujimoto et al. (2005), and time averaging has no effect on the $E W$ if the signal is strongly concentrated in a single flare. This source appears much like a "text book" example of fluorescent emission originating from photoionization both with respect to temporal behavior and $E W$.

\subsubsection{COUP 1040}

COUP 1040 remains virtually invisible in the $>6.2 \mathrm{keV}$ band, except for a flare period during $\mathrm{O} 2$. Figure $3 \mathrm{G}$ shows the light curves. The LLC indicates fluorescent emission accompanying a flare. There is a steep rise in a short $(\approx 10 \mathrm{ks})$ interval in the central flare, and the CLLC continues to rise during the decay phase. Our automated analysis determines an interval containing the decay phase (266-544 ks) of the flare to be the most promising candidate interval to be searched for fluorescent $\mathrm{Fe} \mathrm{K}_{\alpha}$ I emission. In Fig. 9 (left panel) we show the spectrum pertaining to this interval. Indeed, there may be excess emission at $6.4 \mathrm{keV}$; however, the continuum in the $5.2-6.2 \mathrm{keV}$ range is not well fitted by the model, so that the CLLC may be misleading in this case.

The right hand panel of Fig. 9 shows the spectrum during the $\mathrm{O} 2$, which covers the flare and its decay phase. The signal is much stronger here, and the fluorescent line is detectable. While the line flux of $11(6-20) \times 10^{-7} \mathrm{ph}\left(\mathrm{cm}^{2} \mathrm{~s}\right)^{-1}$ is considerably higher than the value reported by Tsujimoto et al. (2005), our $E W$ of $178(90-310) \mathrm{eV}$ is compatible with their result, as it 

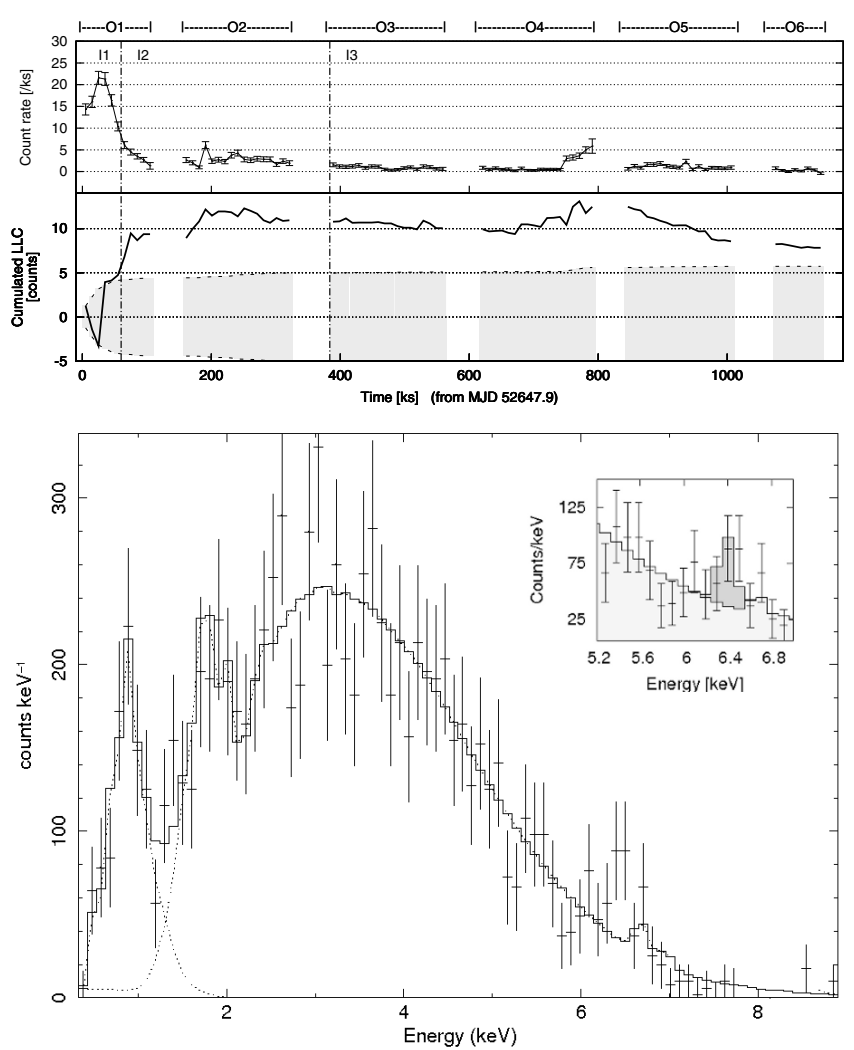

Fig. 10. Upper panel: the $2-9 \mathrm{keV}$ band light curve and the CLLC of COUP 267. Lower panel: the spectrum during the 30-196 ks interval with a close-up of the $\mathrm{Fe} \mathrm{K}_{\alpha} \mathrm{I}$ line region.

remains unchanged by time averaging if no further signal is accumulated.

\subsection{Three individual sources with distinct behavior}

In the following section we present three additional individual sources with a detection of fluorescent emission. The sources show three different types of temporal behavior of the $\mathrm{Fe} \mathrm{K}_{\alpha} \mathrm{I}$ line, and further demonstrate the variety of $\mathrm{Fe} \mathrm{K}_{\alpha} \mathrm{I}$ line emitters.

\subsubsection{COUP 267}

The X-ray spectrum of COUP 267 shows the characteristics of a "two-absorber X-ray source" (TAX source, e.g., Güdel et al. 2007), with a mildly absorbed soft component dominating below about $1 \mathrm{keV}$ and a hard component hidden behind a significantly higher column of material. During the leading $100 \mathrm{ks}$ of COUP campaign data, COUP 267 shows a flare (see Fig. 10, upper panel). A comparison of the soft (0.3-1.2 keV) light curve and its hard counterpart $(1.2-9.0 \mathrm{keV})$ uncovers that the flare exclusively affects the hard band.

In Fig. 10 we show the light curves and the spectrum of COUP 267 during the 30-196 ks time span selected by our automatic search for fluorescence, which basically covers the decay phase of the flare. The $E W$ of the fluorescent line amounts to $400(180-670) \mathrm{eV}$ here. The two differently absorbed thermal components can be clearly distinguished in the spectral model, and excess emission at the energy of the fluorescent line is visible. The upper right hand box in Fig. 10 shows a close-up of this region, where the best-fit line is also indicated.
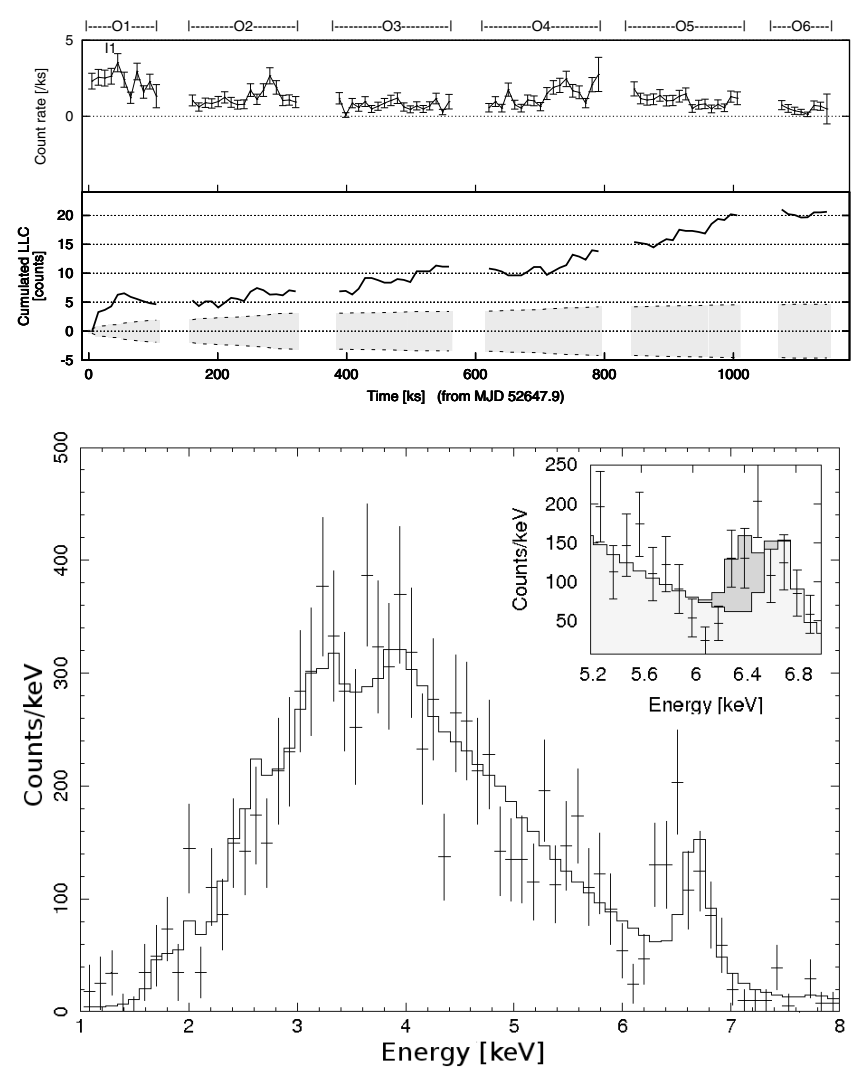

Fig. 11. Upper panel: the $2-9 \mathrm{keV}$ band light curve and the CLLC of COUP 599. Lower panel: the spectrum in the 10-1080 ks interval and a close-up of the $\mathrm{Fe} \mathrm{K}_{\alpha} \mathrm{I}$ line region.

The TAX nature of this source implies a considerable amount of circumstellar material surrounding the central star, where fluorescent emission may well have its origin. Although the measured $E W$ appears higher than the model predictions (see Sect. 6), the fluorescent emission is associated with the flare, and we conclude that photoionization provides a reasonable explanation for the observation.

\subsubsection{COUP 599}

The strength of the X-ray source COUP 599 remains constant through the entire COUP campaign as demonstrated by the light curve shown in Fig. 11. The CLLC rises almost without interruption. Accordingly, the most promising fluorescent interval found in our automated search comprises almost all data.

In the lower panel of Fig. 11, we show the spectrum of the source in the $10-1080 \mathrm{ks}$ interval. There is excess emission at $6.4 \mathrm{keV}$, which we attribute to the fluorescent line, and we determine an $E W$ of $450(220-660)$ eV for it. The position of COUP 599 is about 1 arcsec northwest of the BecklinNeugebauer object (Becklin \& Neugebauer 1967), and as already noted by Garmire et al. (2000) in a pre-COUP study, the $\mathrm{X}$-ray source may be related to it, although an independent origin cannot be excluded.

The X-ray luminosity of this source is constant and so is its Fe $\mathrm{K}_{\alpha}$ I line flux. Again, the $E W$ is higher than typical values derived by Drake et al. (2008), but the strong absorption with a column density of $N_{\mathrm{H}}=7.6(6.6-8.7) \times 10^{22} \mathrm{H} \mathrm{cm}^{-2}$, points towards the presence of circumstellar material, which could yield quite favorable geometry. 

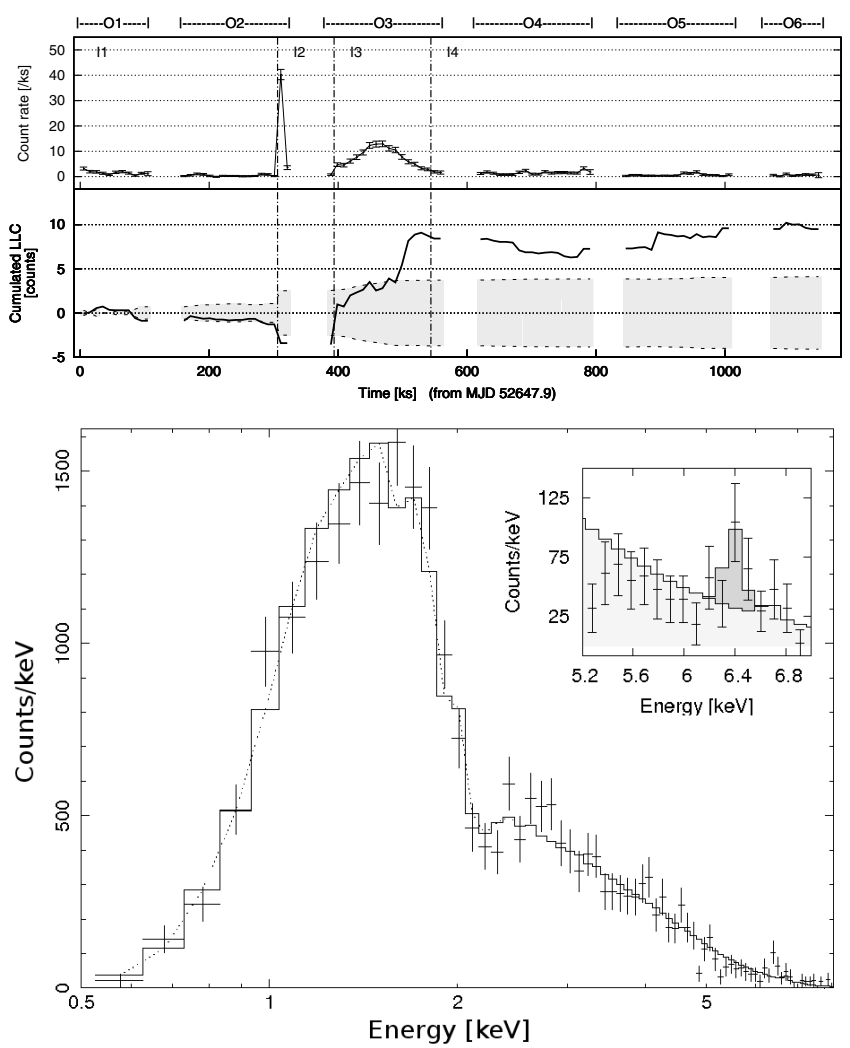

Fig. 12. Upper panel: the $2-9 \mathrm{keV}$ band light curve and the CLLC of COUP 1499. Lower panel: the spectrum in the 384-524 ks interval and a close-up of the $\mathrm{Fe} \mathrm{K}_{\alpha}$ I line region.

\subsubsection{COUP 1499, V NX Ori}

While V NX Ori is found in quiescence for the longest part of the COUP campaign, it also shows a period of remarkable activity. This begins with a very short flare taking place towards the end of the second observation. Here, the count rate skyrockets to about $40 \mathrm{c} \mathrm{ks}^{-1}$ (2-9 keV band) within $2 \mathrm{ks}$ and then decays quickly, so that the entire event does not last for more than $\approx 15 \mathrm{ks}$.

The next observation starts with another short flare that shades into a long-lasting, possibly related flare event. In Fig. 12 we show the spectrum during the 384-524 ks time span found by our automated analysis (see Table 2), which approximately covers the second, long-lasting flare.

While the CLLC does not indicate fluorescent emission during the first, short, and more violent event, it does during the longer flare. The associated spectrum demonstrates line emission for which we measure an $E W$ of $370(145-630) \mathrm{eV}$.

\subsection{Consequences of temporal resolution on the Fe $\mathrm{K}_{\alpha}$ I line equivalent widths}

In the preceding passages we demonstrated the temporal behavior of the $\mathrm{Fe} \mathrm{K}_{\alpha}$ I line for a number of sources including those presented by Tsujimoto et al. (2005). The $E W$ of the $\mathrm{Fe}_{\alpha} \mathrm{I}$ line is often used to check whether the observations can be reconciled with predictions from theory. Therefore, it is worth briefly discussing the effects of the time resolution on this quantity.

In sources where one major flare provides the majority of all source photons, including $\mathrm{Fe} \mathrm{K}_{\alpha}$ I line photons, such as COUP 649 and COUP 1030, time averaging over a wider span has no effect on the calculated $E W$, because line and continuum emission are both scaled by the same factor. It does, however, have an effect on the measured fluxes, which are then too low.

The measured $E W$ will be too low if the quiescent phases, which are usually much longer than active periods, or several flares contribute a significant amount of flux, as for instance in COUP 331 and COUP 621. In these cases, continuum signal is accumulated, which, afterwards, is compared to the $\mathrm{Fe} \mathrm{K}_{\alpha} \mathrm{I}$ line signal being collected during a shorter period. Here, both the measured average $\mathrm{Fe} \mathrm{K}_{\alpha}$ I line flux and the associated $E W$ will be lower than values determined for appropriate time spans.

As it is impossible to accumulate negative signal in the $\mathrm{Fe} \mathrm{K}_{\alpha} \mathrm{I}$ line or the underlying continuum, maybe with the exception of background and statistical fluctuations, the time-averaged values can be regarded as a lower limit on both the $\mathrm{Fe} \mathrm{K}_{\alpha} \mathrm{I}$ line flux and $E W$.

\subsection{Combined excess emission}

In the previous sections, we have presented a sample of sources with excess emission at $6.4 \mathrm{keV}$, which we attribute to the fluorescent $\mathrm{Fe}_{\alpha} \mathrm{I}$ line. In this section we combine a larger amount of data to further corroborate this statement.

In Fig. 13 we show the summed residuals corresponding to all intervals listed in Table 2 in the upper panel and, in the lower one, the summed residuals for all sources showing no fluorescent emission according to our analysis. For each spectrum we determined the residuals by subtracting the best-fit model from the observed data and added up the individual results. We prefer this approach to adding up the individual spectra themselves, because these differ significantly from each other both in flux level and spectral shape. The residuals already account for the differences in spectral shape, and the contribution of spectra with more photons grows with the square root of the photon number, and not linearly as would be the case otherwise.

An emission line feature, which is not accounted for in the models but present in some or all of the spectra, should lead to an accumulation of residual photons at the respective energy. In Fig. 13 we show the result using the residuals with respect to the thermal model (cf. Sect. 4.3) and a power-law model extended by a Gaussian line of variable width (but $<0.1 \mathrm{keV}$ ) and normalization at a fixed energy of $6.7 \mathrm{keV}$ to account for the hot iron lines.

In both cases, an excess at $6.4 \mathrm{keV}$ can be clearly identified. We find that this excess is represented well by a Gaussian centered at $6.4 \mathrm{keV}$. The best-fit width of the Gaussian is $100 \mathrm{eV}$, which is consistent with an intrinsically narrow, instrumentally broadened line at the location of the fluorescent $\mathrm{Fe} \mathrm{K}_{\alpha} \mathrm{I}$ line. Leaving the central energy as a free fit-parameter does not alter this result. Depending on the considered energy range for adapting the Gaussian and on whether we allow an offset for the Gaussian line, we find amplitudes of 300-400 photons. Therefore, we argue for the presence of $\approx 350$ excess photons, which can be attributed to the fluorescent $\mathrm{Fe} \mathrm{K}_{\alpha} \mathrm{I}$ line in these spectra. This number can be converted into an average line $E W$ of roughly $250 \mathrm{eV}$, which we find in good agreement with previous results.

Instead of collecting the residuals only from the periods of bona-fide fluorescent emission, the collection can also be inverted to comprise only data of those sources in which no $\mathrm{Fe}_{\alpha} \mathrm{I}$ line emission was detected. Neither of the resulting curves, whether calculated with respect to either the thermal or the power law-models, shows a distinct feature at $6.4 \mathrm{keV}$ (Fig. 13, lower panel). The residuals collected from the thermal models show a substructure indicating that the average spectral 


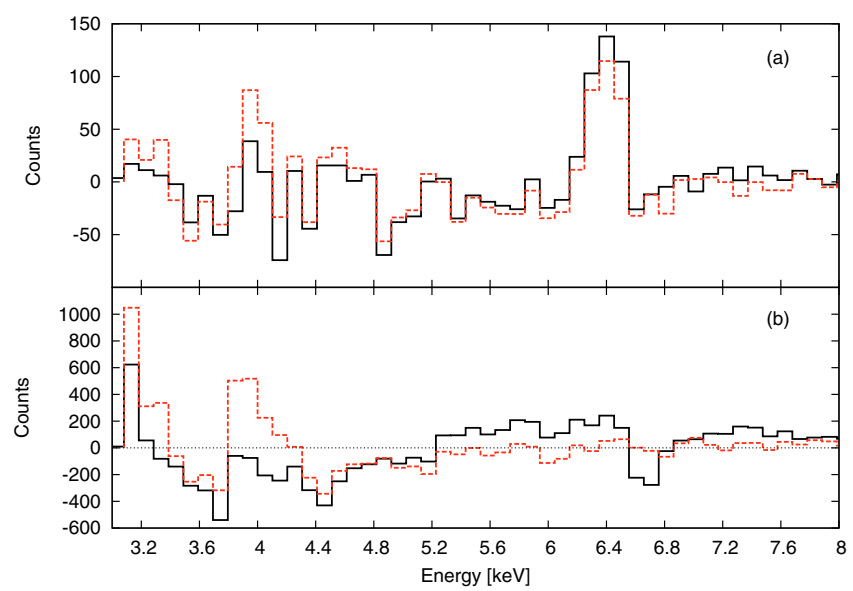

Fig. 13. The summed residuals from all bona-fide fluorescent intervals (panel a)) and the same for all sources not showing intervals with fluorescent emission (panel b)). Solid (black) line: the residuals with respect to thermal models, dashed (red) line: the residuals with respect to a power-law model with an additional hot iron line at $6.7 \mathrm{keV}$.

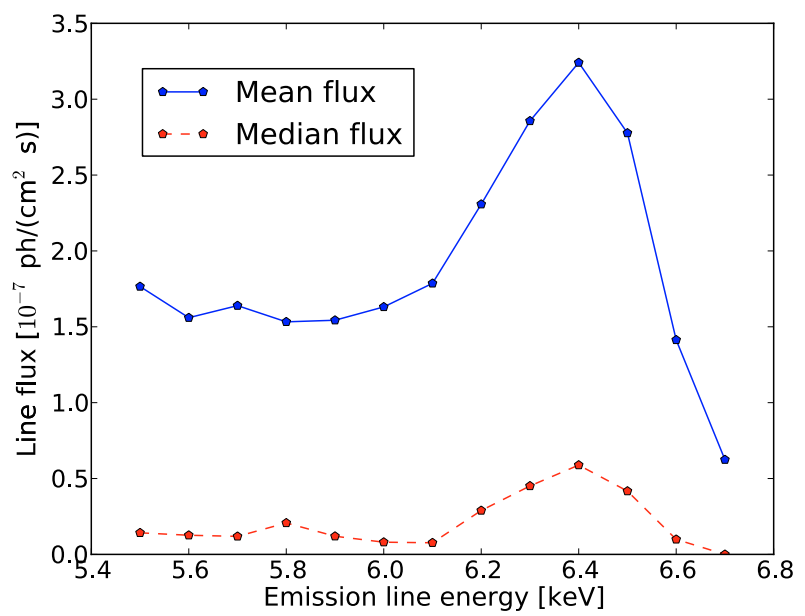

Fig. 14. Mean (solid line) and median (dashed line) flux for narrow emission line at various energies.

slope is too steep. Such an effect is not present in the powerlaw models, which, however, cover much less of the spectrum. After consulting the power-law fit residuals, a small excess of $\approx 100$ photons at $6.4 \mathrm{keV}$ may still be present, but it is not clearly set apart from the noise. We also find no surplus of negative residuals here as would be expected, if we had only removed sources showing a positive noise excess from the sample.

\subsection{Comparison features at different energies}

For the following analysis, we revert to the Bayesian block data, i.e., a subdivision made without paying special attention to the presence or absence of the $\mathrm{Fe} \mathrm{K}_{\alpha} \mathrm{I}$ line, even though this might be the case due to physical reasons. Starting from the best-fit thermal model, we add a narrow emission line at various energies between $5.5 \mathrm{keV}$ and $6.7 \mathrm{keV}$. For each Bayesian block, we record the best-fit line fluxes and $E W \mathrm{~s}$.

In Fig. 14 we show the mean and median line flux determined for the emission line, shifting it from $5.5 \mathrm{keV}$ to $6.7 \mathrm{keV}$ in steps of $100 \mathrm{eV}$. This set-up includes the actual $\mathrm{Fe} \mathrm{K}_{\alpha} \mathrm{I}$ line at $6.4 \mathrm{keV}$ as a special case. Both mean and median line flux are $>0$ everywhere, since we demand an emission line. We do not expect any strong features between $5.5 \mathrm{keV}$ and $6.3 \mathrm{keV}$, so that the results can be attributed to the photon noise.

The mean line flux shown in Fig. 14 remains constant until the line energy exceeds $6.1 \mathrm{keV}$, after which the mean flux rises, reaches a maximum at $6.4 \mathrm{keV}$, and falls again. The decrease towards $6.7 \mathrm{keV}$ happens because the thermal models include the hot iron lines. To assure that only a few high values do not dominate the plot, which is conceivable if the mean line flux is considered, we also give the median line flux, which is more stable against outliers. At a lower level, the median flux shows the same basic pattern with a maximum at $6.4 \mathrm{keV}$. The level shows that many values are close to zero, reflecting the cases in which an absorption line would formally yield a better fit.

At each line energy the statistic for the comparison line is different, because it is affected by the spectral shape and the effective area. Under the hypothesis that there is nothing special about the $\mathrm{Fe}_{\alpha}$ I line energy, so that the majority of our detections should actually be attributed to noise, the derived result is implausible. If this hypothesis was to be accepted, there should be a gradual evolution of the mean and median line fluxes over the entire range, which is indeed the case until the line energy exceeds $6.1 \mathrm{keV}$, but not beyond.

The findings summarized in Fig. 14 are compatible with an instrumentally broadened emission line at $6.4 \mathrm{keV}$, and we adduce that this is a corroboration of our interpretation of the observed excess emission as fluorescent $\mathrm{Fe}_{\alpha}$ I line emission, which is distinct from photon noise.

\subsection{Duty cycle of Fe $\mathrm{K}_{\alpha}$ I line emission}

In Table 2 we list the sources and time spans that show significant $\mathrm{Fe}_{\alpha} \mathrm{I}$ line emission according to our analysis. The table comprises 23 sources, with a total observation time of $23 \times 838 \mathrm{ks} \approx 19000 \mathrm{ks}$. Summing up the interval lengths, we find that fluorescent emission is detected during $\approx 50 \%$ of this time.

Using the entire sample of 106 sources as a reference, fluorescent emission is detectable during about $10 \%$ of the observation time, and it is distributed among $20 \%$ of the sample sources. This consideration only takes into account those sources in which fluorescent emission can be claimed with reasonable certainty. Based on the creation scenario for fluorescent emission, it seems likely that there are more fluorescent sources, yet a detection cannot be claimed where the signal remains insufficient. The duty cycle derived here should, therefore, be regarded as a minimum, limited by the sensitivity of the instrument and, of course, by our method.

\section{Origin of the $\mathrm{Fe} \mathrm{K}_{\alpha}$ I line emission}

The reality of the fluorescent $\mathrm{Fe} \mathrm{K}_{\alpha} \mathrm{I}$ line has so far been established in several stellar sources. While all authors agree that neutral or weakly ionized iron gives rise to the photons making up this spectral component, there is still some debate whether nonthermal electrons contribute to the excitation of the iron ions. In the following, we briefly juxtapose photoionization and electron collisional excitation regarding the expected $E W \mathrm{~s}$ and temporal behavior.

\subsection{Implications of photoionization}

The fluorescent emission caused by photoionized neutral or weakly ionized iron was investigated theoretically by a number 
of authors. Bai (1979) and Drake et al. (2008) carried out Monte Carlo calculations, simulating solar and stellar flares. George \& Fabian (1991) simulated an accretion disk illuminated with a power-law spectrum approaching the situation in AGN sources. George \& Fabian (1991) obtained typical EWs between 100-200 eV for a centrally illuminated disk using power-law photon-indices between $\Gamma=1.3-2.3$. The $E W \mathrm{~s}$ obtained by Bai (1979) and Drake et al. (2008) for thermal input spectra tend be smaller, rarely exceeding $100 \mathrm{eV}$.

While the above results refer to solar iron abundances of $\approx 4 \times 10^{-5} \mathrm{Fe} / \mathrm{H}$, the $\mathrm{Fe} \mathrm{K}_{\alpha} \mathrm{I}$ line $E W$ caused by photoionized iron can be increased, when larger iron abundances are assumed (Drake et al. 2008). In an analysis of the correlation between iron abundance and $\mathrm{Fe} \mathrm{K}_{\alpha}$ I line $E W$, however, Ballantyne et al. (2002) found that the $E W$ cannot be increased beyond any limit. As already indicated by Bai (1979) (Fig. 4) or Matt et al. (1997), the $E W$ rises linearly for iron abundances close to or lower than the solar value, beyond which the relation flattens. According to Ballantyne et al. (2002), the maximal obtainable Fe $\mathrm{K}_{\alpha} \mathrm{I}$ line $E W$ is around $800 \mathrm{eV}$.

The $\mathrm{Fe} \mathrm{K}_{\alpha} \mathrm{I}$ line $E W \mathrm{~s}$ expected from photoionized iron is, thus, $\approx 100 \mathrm{eV}$ and may reach $\approx 800 \mathrm{eV}$ under very favorable conditions. The light curve of the $\mathrm{Fe} \mathrm{K}_{\alpha}$ I line should be correlated with the light curve of the incident soft X-ray continuum, but reverberation effects may be present. Assuming a typical scale of $20 \mathrm{AU}$ for the extent of a stellar source, delays, if present, would be $\approx 10 \mathrm{ks}$.

High $\mathrm{Fe}_{\alpha} \mathrm{I}$ line $E W \mathrm{~s}$ and a virtually unpredictable temporal behavior may be obtained, if totally or partially hidden X-ray sources are considered, as proposed by Drake et al. (2008). The continuum emission by those sources can contribute to the reprocessed photon field, but their direct continuum radiation remains invisible, so that the measured $\mathrm{Fe} \mathrm{K}_{\alpha}$ I line flux may be significant, providing a large $E W$, which appears to be uncorrelated with the measured continuum.

\subsection{Electron collisional excitation}

The subject of electron collisional excitation of the fluorescent $\mathrm{Fe} \mathrm{K}_{\alpha} \mathrm{I}$ line was, for example, investigated by Emslie et al. (1986). These authors calculated the $\mathrm{Fe} \mathrm{K}_{\alpha} \mathrm{I}$ line photon flux, $\Phi$, due to electron excitation, and the result is given in their Eq. (12). In combination with their Eq. (7), the relation can be recast and written as

$$
\frac{4 \pi R^{2}}{A F\left(>E_{1}\right)} \Phi=\omega \beta\left(A b_{\mathrm{Fe}}\right) \frac{\gamma-1}{\gamma} \frac{E_{1}^{\gamma-1}}{K v} \int_{\chi}^{\infty} E^{1-\gamma} Q_{I}(E) \mathrm{d} E
$$

where $A b_{\mathrm{Fe}}$ is the iron abundance, $\gamma+1$ is the spectral index of the incident electron power law spectrum, $E_{1}$ the lower cut-off energy of the electron spectrum, $K=2.61 \times 10^{-18} \mathrm{keV}^{2} \mathrm{~cm}^{-2}$, $v=1.6 \times 10^{-9}$ is the conversion factor from $\mathrm{keV}$ to erg, $\chi=7.112 \mathrm{keV}$ is the $\mathrm{K}$-shell ionization energy of neutral iron, $\omega$ is the iron fluorescence yield, $\beta$ the branching between $\mathrm{K}_{\alpha}$ and $\mathrm{K}_{\beta, \gamma \ldots}, Q_{I}$ the cross section for $\mathrm{K}$-shell ionization by iron (see e.g., Hombourger 1998), $R$ the distance to the source, $F\left(>E_{1}\right)$ the total injected electron energy flux in $\mathrm{erg} /\left(\mathrm{s} \mathrm{cm}^{2}\right)$, and $A$ the area of the flare. In Eq. (13), we have already assumed that there is no hydrogen column between the electron injection site and the neutral iron, which corresponds to $N^{*}=0$ in Emslie et al. (1986).
Assuming $\gamma=2.1, A b_{\mathrm{Fe}}=4 \times 10^{-5}, E_{1}=20 \mathrm{keV}$, and a distance of $450 \mathrm{pc}$, Eq. (13) can be partially evaluated as

$A F\left(>E_{1}\right) \approx 4.5 \times 10^{41} \frac{\mathrm{cm}^{2} \text { erg }}{\text { photon }} \times \Phi$,

where $A F\left(>E_{1}\right)$ is the incident energy flux in the form of electrons in erg s $\mathrm{s}^{-1}$. Using Eq. (14) we derive that, for the typical Fe $\mathrm{K}_{\alpha}$ I line fluxes of several times $10^{-7} \mathrm{ph}\left(\mathrm{cm}^{2} \mathrm{~s}\right)^{-1}$ measured for the COUP sources (Tsujimoto et al. 2005), the injected flux of electrons in excess of $20 \mathrm{keV}$ should be on the order of $10^{35} \mathrm{erg} \mathrm{s}^{-1}$.

This number is enormous compared to solar values, where the total energy deposited in nonthermal electrons during a flare is of the order of $10^{32} \mathrm{erg}$. Even though flares in Orion YSOs may be "several orders of magnitude more powerful than any solar flare" (Getman et al. 2008), the energetics remain problematic. Using Eq. (17) from Saint-Hilaire \& Benz (2005), we estimate the amount of thermal energy, $E_{\mathrm{th}}$, contained in the plasma of a large flare to be

$E_{\mathrm{th}}=3 k_{b} T \sqrt{E M^{2} / n^{2} \cdot f} \approx 10^{36} \mathrm{erg}$,

assuming an emission measure of $10^{55} \mathrm{~cm}^{-3}$, a density of $n=$ $10^{12} \mathrm{~cm}^{-3}$, a temperature of $k_{b} T=10 \mathrm{keV}$, and a filling factor, $f$, of unity. In their analysis of RHESSI data of medium sized solar flares, Saint-Hilaire \& Benz (2005) conclude that $E_{\text {th }}$ is close to the energy contained in nonthermal particles. Extrapolating this result to the Orion flares, we conclude that currents strong enough to provide significant $\mathrm{Fe}_{\alpha} \mathrm{I}$ line fluxes cannot be sustained for longer than a few seconds.

As pointed out by Emslie et al. (1986), a contribution of electron induced fluorescent emission may be observed during the impulsive phase of a flare. The $\mathrm{Fe} \mathrm{K}_{\alpha} \mathrm{I}$ line flux due to electron collisional excitation should be temporally correlated with the hard X-ray flux, which was, indeed observed by Emslie et al. (1986). Such data are, however, not available for Orion, so that we cannot revert to this line of argumentation.

Recently, Peterson et al. (2010) presented high-resolution radio images of the Algol binary system resolving a large coronal loop reaching from one to the other pole of the $\mathrm{K}$ giant secondary, being orientated towards the primary B star. The authors modeled the radio emission observed from this loop by assuming synchrotron emission from an electron population with a homogeneous density of $n_{\mathrm{e}}=10^{-3}$ electrons $/ \mathrm{cm}^{-3}$ and a power-law spectrum so that $n(E)=n_{\mathrm{e}}\left(E / E_{\min }\right)^{-3 / 2}$ where $E_{\min }=80 \mathrm{keV}$. Assuming that the electrons are moving at the speed of light, we estimate an electron flux of roughly $3 \times 10^{10} \mathrm{~cm} \cdot 1000 \mathrm{~cm}^{-3}=$ $3 \times 10^{13}$ electrons $/ \mathrm{cm}^{-2}$. When also assuming a loop length of $10^{12} \mathrm{~cm}$ and a radius-to-loop length ratio of 0.1 (Favata et al. 2005), the electron flux over the entire loop cross section becomes $\approx 10^{35}$ electrons $/ \mathrm{cm}^{2}$. If we attribute an energy of $100 \mathrm{keV}$ to all of these electrons, the resulting energy flux in the form of energetic electrons becomes $10^{28} \mathrm{erg} \mathrm{s}^{-1}$. Although this number is still far below the needed value, we note that Favata et al. (2005) find evidence of large magnetic loops with half lengths of $\approx 10^{12} \mathrm{~cm}$ in their analysis of large COUP flares, which may connect the stellar photosphere with a disk. Although unable to give a detailed physical modeling, we do not exclude that under certain conditions sufficient fluxes of energetic electrons may be sustained in large magnetic structures.

When a population of high-energy electrons hits a thick target such as a stellar photosphere, the electrons thermalize in the plasma and heat it. This gives rise to the evaporation of soft 
X-ray emitting loops observed during solar and stellar flares. Inspired by their findings, Giardino et al. (2007) elaborate on a mechanism that can prevent a population of accelerated electrons from causing a soft X-ray flare. Basically, the authors suggest that, if the plasma density in the flux tube into which the electrons are injected is high enough, the electrons do not thermalize in the photosphere, but are decelerated in situ. During this process, energy is deposited in nonthermal hard X-rays and collisional excitation of heavier ions such as iron, potentially resulting in $\mathrm{Fe} \mathrm{K}_{\alpha}$ I line photons. Appropriate conditions for this mechanism to work may, indeed, be found in young stars with ongoing accretion.

\subsection{Putting our analysis into context}

As outlined in Sect. 6, model calculations of the $\mathrm{Fe} \mathrm{K}_{\alpha} \mathrm{I}$ line strength based on photoionization rarely predict line $E W \mathrm{~s}>$ $100 \mathrm{eV}$. Yet, the $E W \mathrm{~s}$ listed in Table 2 tend to be significantly more than $100 \mathrm{eV}$, even though some may be smaller considering the errors. The values reported in Table 2 are qualitatively compatible with the numbers published for Elias 29, for which Giardino et al. (2007) derive $E W$ s of up to $\approx 800 \mathrm{eV}$ during their second time segment.

If photoionization is to be held responsible for the line emission, it must explain all of these observations: both the observed $E W$ and the temporal behavior. Since the observed $E W$ s tend to be higher than typical predictions, this issue should be addressed first. Drake et al. (2008) discuss four ways in which the Fe $\mathrm{K}_{\alpha} \mathrm{I}$ line $E W$ may be raised. These are supersolar Fe abundances, disk flaring, a contribution of electron collisional excitation, and line-of-sight obscuration of the central flaring source. According to Drake et al. disk flaring, which increases the solid angle coverage of the reflector, can increase the expected $E W$ by factors $<2$, and increasing the iron abundance leads to a roughly linear response of the line $E W . E W \mathrm{~s}$ on the order of $400 \mathrm{eV}$ as in the majority of our detections, are, therefore, within reach without reverting to extreme physical conditions. Additionally, we note that obscuration of the X-ray source does not only increase the $\mathrm{Fe}_{\alpha} \mathrm{I}$ line $E W$ when the absorber is impenetrable, but also if it only has small optical thickness. The reason is that fluorescent photons at $6.4 \mathrm{keV}$ can be created as the X-rays are passing the absorber, while continuum photons can only be destroyed. Yaqoob et al. (2010) show, in a different context, that the optically thin approximation for the fluorescence problem breaks down for column densities as low as $\approx 2 \times 10^{22} \mathrm{H} \mathrm{cm}^{-2}$, which should also be the scale on which this effect may become noticeable. The effect may well be responsible for a fraction of the observed $E W$ in sources with large enough absorbing columns, i.e., in about half of our sources (see Table 2).

The most extreme $\mathrm{Fe}_{\alpha}$ I line is observed during the rise phase of a flare in V 1486 Ori, where we determine a line $E W$ of $\approx 1400 \mathrm{eV}$ (see Sect. 5.1.1) exceeding typical model predictions by an order of magnitude. To explain this measurement in the frame of photoionization, Drake et al. (2008) suggest that the $\mathrm{Fe} \mathrm{K}_{\alpha} \mathrm{I}$ line stems from photoionization by a partially hidden $\mathrm{X}$-ray source. While this is an appealing idea, the LLC shown in Fig. 4 also suggests an impulsive peak, as presented by Emslie et al. (1986) (Fig. 3) for the case of the Sun. According to Emslie et al. (1986), this peak, which, however, lasted for no longer than $\approx 20 \mathrm{~s}$ there, is associated with $\mathrm{Fe} \mathrm{K}_{\alpha}$ I line emission due to electron collisional excitation. Assuming that this is also true for V 1486 Ori, we can calculate the electron flux needed to excite the observed line. Applying Eq. (14) and substituting the line flux of $\approx 8 \times 10^{-6} \mathrm{ph}\left(\mathrm{cm}^{2} \mathrm{~s}\right)^{-1}$ during the time span given in Table 2 yields an energy input of about $3 \times 10^{36} \mathrm{ergs} \mathrm{s}^{-1}$, which would be required to sustain this line by electron collisional excitation. Whether the observed $\mathrm{Fe} \mathrm{K}_{\alpha}$ I line emission of V 1486 Ori contains a contribution coming from electron collisional excitation cannot ultimately be decided from the data at hand, but if is does, the energy released into electrons must be enormous.

Even though $E W \mathrm{~s}$ of $\approx 400 \mathrm{eV}$ may be explained by photoionization, some sources still show puzzling temporal behavior. For example COUP 621, one of the Tsujimoto et al. (2005) sources, shows two flares, which do not seem to be accompanied by fluorescent emission, whereas $\mathrm{Fe}_{\alpha} \mathrm{I}$ line emission is observed during a bona-fide quiescent period preceding the first flare. This observation may be attributed to a "hidden flare" somewhere behind the star or the disk, which is only visible in its reprocessed light. It may also be associated with an event preceding and maybe preparing the first flare, such as magnetic reconnection releasing high-energy electrons. Feigelson et al. (1998) show that there are large-scale magnetic structures in an $\mathrm{X}$-ray protostar in Corona Australis and that the observed polarized radio emission arises from a nonthermal electron population spiraling along the field lines. Giardino et al. (2007) argue that magnetic reconnection can be sustained for several days, but they do not give a physical model for it. As the energetics of collisional electron excitation is very challenging (see Eq. (14)), however, this explanation seems physically problematic, and, in light of current knowledge, photoionization by a hidden flare appears a more straightforward answer. Similar to COUP 621, other sources like V 1486 Ori and V NX Ori show flares both with and without detectable fluorescent emission. We interpret this as a relic of a manifold geometry. Depending on the location of flare eruption, the conditions for photoionizing iron and creating fluorescent photons, at least towards our line of sight, can be quite different. Perhaps some flares are associated with large magnetic structures connecting photosphere and disk, as proposed by Giardino et al. (2007) or Favata et al. (2005), but also different locations above the photosphere itself have considerable consequences for the observed $\mathrm{Fe}_{\alpha} \mathrm{I}$ line $E W$ (e.g., Bai 1979; Drake et al. 2008).

\section{Conclusion}

As a first result of this work, we state that there are far more $\mathrm{X}$-ray sources with detectable fluorescent $\mathrm{Fe} \mathrm{K}_{\alpha} \mathrm{I}$ line emission in the COUP field than previously reported. While it extends the sample of known stellar Fe $\mathrm{K}_{\alpha} \mathrm{I}$ line sources, the major part of this study is dedicated to analyzing of the temporal behavior of the $\mathrm{Fe} \mathrm{K}_{\alpha}$ I line emission, which yields another result: strong diversity in the relation between $\mathrm{Fe} \mathrm{K}_{\alpha} \mathrm{I}$ line emission and soft $\mathrm{X}$-ray continuum.

Fluorescent emission is found to accompany a flare in some cases, sometimes it is a persistent feature, and in other cases it appears "out of nothing" with no obvious response by the soft $\mathrm{X}$-ray continuum. The diverse temporal behavior of fluorescent $\mathrm{Fe}_{\alpha}$ I line emission cannot always be easily reconciled with the picture of photoionization as the dominant excitation mechanism of this emission. Even in sources that show flares during the COUP campaign, the majority of fluorescent photons may still be observed in bona-fide quiescent phases. In those cases, but also in others, the observed $E W \mathrm{~s}$ are often appreciably higher than predicted by photoionization-based model calculations that apply conventional assumptions on geometry and elemental abundances. 
Nonetheless, we argue that photoionization remains the most promising explanation for the observations. The alternative mechanism of electron collisional excitation is appealing in some cases, because it provides an explanation for $\mathrm{Fe} \mathrm{K}_{\alpha} \mathrm{I}$ line emission not accompanied by a change in the level of soft X-ray emission, but the energetics are challenging. To explain the observed $\mathrm{Fe} \mathrm{K}_{\alpha} \mathrm{I}$ line fluxes, enormous fluxes of energetic electrons, which otherwise remain invisible, must sometimes be sustained for days. Electron collisional excitation may certainly provide a fraction of the fluorescent line photons, but it can hardly explain the majority.

The observations can be reconciled with predictions based on photoionization, if we assume more complicated environments. This includes partially or completely hidden X-ray sources as well as modification of the iron abundance. Also, fluorescence occurring in the circumstellar absorbing material, currently not accounted for in the models, possibly accounts for (a fraction of) the observed $E W \mathrm{~s}$.

Our current understanding of the fluorescent $\mathrm{Fe} \mathrm{K}_{\alpha} \mathrm{I}$ line is certainly unsatisfactory, and the great diagnostic power of the line for a variety of celestial sources makes further observations clearly desirable.

Acknowledgements. This work made use of data obtained from the Chandra data archive. S.C. acknowledges support from the DLR under grant 50 OR0105. We thank Eric D. Feigelson for providing us with the outcomes of their analysis of the COUP data.

\section{References}

Anders, E., \& Grevesse, N. 1989, Geochim. Cosmochim. Acta, 53, 197 Bai, T. 1979, Sol. Phys., 62, 113

Ballantyne, D. R., \& Fabian, A. C. 2003, ApJ, 592, 1089
Ballantyne, D. R., Fabian, A. C., \& Ross, R. R. 2002, MNRAS, 329, L67 Bambynek, W., Crasemann, B., Fink, R. W., et al. 1972, Rev. Mod. Phys., 44, 716

Becklin, E. E., \& Neugebauer, G. 1967, ApJ, 147, 799

Cash, W. 1979, ApJ, 228, 939

Czesla, S., \& Schmitt, J. H. H. M. 2007, A\&A, 470, L13

Doschek, G. A., Meekins, J. F., Kreplin, R. W., Chubb, T. A., \& Friedman, H. 1971, ApJ, 170, 573

Drake, J. J., Ercolano, B., \& Swartz, D. A. 2008, ApJ, 678, 385

Emslie, A. G., Phillips, K. J. H., \& Dennis, B. R. 1986, Sol. Phys., 103, 89

Favata, F., Flaccomio, E., Reale, F., et al. 2005, ApJS, 160, 469

Feigelson, E. D., Carkner, L., \& Wilking, B. A. 1998, ApJ, 494, L215

Garmire, G., Feigelson, E. D., Broos, P., et al. 2000, AJ, 120, 1426

George, I. M., \& Fabian, A. C. 1991, MNRAS, 249, 352

Getman, K. V., Flaccomio, E., Broos, P. S., et al. 2005, ApJS, 160, 319

Getman, K. V., Feigelson, E. D., Broos, P. S., Micela, G., \& Garmire, G. P. 2008, ApJ, 688, 418

Giardino, G., Favata, F., Pillitteri, I., et al. 2007, A\&A, 475, 891

Güdel, M., Telleschi, A., Audard, M., et al. 2007, A\&A, 468, 515

Hombourger, C. 1998, J. Phys. B Atom. Mol. Phys., 31, 3693

Imanishi, K., Koyama, K., \& Tsuboi, Y. 2001, ApJ, 557, 747

Kaastra, J. S., \& Mewe, R. 1993, A\&AS, 97, 443

Matt, G., Fabian, A. C., \& Reynolds, C. S. 1997, MNRAS, 289, 175

Neupert, W. M., Gates, W., Swartz, M., \& Young, R. 1967, ApJ, 149, L79

Parmar, A. N., Culhane, J. L., Rapley, C. G., et al. 1984, ApJ, 279, 866

Peterson, W. M., Mutel, R. L., Güdel, M., \& Goss, W. M. 2010, Nature, 463, 207

Protassov, R., van Dyk, D. A., Connors, A., Kashyap, V. L., \& Siemiginowska, A. 2002, ApJ, 571, 545

Saint-Hilaire, P., \& Benz, A. O. 2005, A\&A, 435, 743

Scargle, J. D. 1998, ApJ, 504, 405

Smith, R. K., Brickhouse, N. S., Liedahl, D. A., \& Raymond, J. C. 2001, ApJ, 556, L91

Sunyaev, R. A., \& Churazov, E. M. 1996, Astron. Lett., 22, 648

Tanaka, Y., Nandra, K., Fabian, A. C., et al. 1995, Nature, 375, 659

Testa, P., Drake, J. J., Ercolano, B., et al. 2008, ApJ, 675, L97

Tsujimoto, M., Feigelson, E. D., Grosso, N., et al. 2005, ApJS, 160, 503

Yaqoob, T., Murphy, K. D., Miller, L., \& Turner, T. J. 2010, MNRAS, 401, 411

Zarro, D. M., Dennis, B. R., \& Slater, G. L. 1992, ApJ, 391, 865 